\title{
Effect of Combination Treatments of Apigenin and Chemotherapy on Apoptosis- Related Genes and Proteins in Acute Leukaemia Cell Lines
}

\author{
Amani A. Mahbub
}

Umm Al-Qura University

Christine L. Le Maitre

Sheffield Hallam University

Neil A. Cross

Sheffield Hallam University

Nicola Jordan-Mahy ( $\square$ n.jordanmahy@shu.ac.uk)

Sheffield Hallam University

\section{Research Article}

Keywords: Acute leukaemia, Apoptosis, Intrinsic/ Mitochondrial Pathway, Extrinsic/ Death Receptor Pathway, Apigenin, Chemotherapy, Etoposide, Cyclophosphamide

Posted Date: November 30th, 2021

DOI: https://doi.org/10.21203/rs.3.rs-1081612/v1

License: (9) (1) This work is licensed under a Creative Commons Attribution 4.0 International License. Read Full License 


\section{Abstract}

Apigenin is a dietary polyphenol found abundantly in fruit and vegetables. It has been shown to sensitize acute lymphoid and myeloid leukaemia cells to topoisomerase inhibitor agents (e.g., etoposide), and alkylating agents (e.g., cyclophosphamide), reducing ATP levels and inducing apoptosis; whilst being protective to control haematopoietic stem cells. This study analysed the expression profiles of intrinsic and extrinsic apoptosis-related genes and proteins to help elucidate the mechanisms of action of apigenin when used in combination with etoposide or cyclophosphamide in acute lymphoid and myeloid leukaemia cells (Jurkat and THP-1). Expression of apoptosis-related genes were measured using a TaqMan ${ }^{\circledR}$ Human Apoptosis Array and the StepOne Plus RT-qPCR System, whilst apoptosis-related proteins were determined using a protein profiler $^{T M}$-human apoptosis array and the LI-COR Odyssey ${ }^{R}$ Infrared Imaging System.

Apigenin when combined with etoposide or cyclophosphamide-induced apoptosis via the mitochondrial pathway, increasing the expression of pro-apoptotic cytochrome $c$, SMAC/DIABLO, and HTRA2/OMI, which promoted caspase- 9 and -3 activation. Targeting anti-apoptotic and/or pro-apoptotic members of the apoptotic pathways is a promising strategy to induce cancer cell death and improve sensitivity to chemotherapy agents.

Here apigenin in combination with etoposide or cyclophosphamide induced apoptosis in leukaemia cells and may have clinical potential in the treatment of leukaemia.

\section{Introduction}

Based on data from the International Agency for Research on Cancer, leukaemia is the tenth most common cause of cancer deaths Worldwide [1]. Leukaemia is a group of life-threatening malignant disorders of the blood and bone marrow, which affects all blood cell lineages and is associated with an increase in the growth rate of blood-forming cells, caused by a failure of programmed cell death pathways and a reduced differentiation of haematopoietic cells [2-4]. Apoptosis plays a crucial role in determining and regulating cellular development, homeostasis, and both physiological and pathological processes [5]. Apoptosis has two core pathways: the intrinsic and the extrinsic pathway [5]. Dysregulation of programmed cell death via apoptosis in leukaemia is common due to abnormal expression or mutation of one or more apoptotic genes, resulting in the survival of malignant cells over their normal counterparts [6-8].

Acute leukaemia is associated with a decrease in cell sensitivity to pro-apoptotic signals by overexpression of BCL-2 family anti-apoptotic proteins such as BCL2, BCLX, and MCL1 [3, 9, 10]; and/or with low levels of pro-apoptotic members such as BAX [11]. This means leukaemic cells can evade apoptosis, and this can lead to chemotherapy resistance, and relapse associated with shorter diseasefree, or overall survival for patients with acute leukaemia's $[3,8,9,11]$. The standard front-line therapeutic approach for acute leukaemia patients is chemotherapy, which consists of multi-agent chemotherapy 
regimens [12-16]. Unfortunately, the multi-agent chemotherapy regimens have a number of off-target effects, which include pancreatitis, coagulopathy, and hepatotoxicity $[14,15]$ and the development of chemoresistance, which contributes to disease relapse, and ultimately patient death [14-17]. It is reported that this is particularly challenging in the treatment of childhood acute lymphocytic leukaemia (ALL), where there is a risk of relapse in $20-30 \%$ of children [18].

Currently, one of the most promising anti-leukaemia strategies is to design targeted therapies with lower toxicities, which reverse chemotherapy-resistance, and improve acute leukaemia patient survival $[3,8]$. Such therapies would induce apoptotic cell death in leukaemia cells, with no or limited collateral damage to normal haematopoietic progenitor cells; and less likely to encounter many of the resistance mechanisms that have been associated with conventional agents [3, 8, 19]. Indeed, Rodriguez et al., 2015 reported that intermediates of apoptosis pathways might be excellent candidates for molecular targeting for cancer treatment [20]. For this reason, it is worth identifying new drugs targeting apoptotic molecules that are abnormally expressed or dysregulated in leukaemic cells; and to restore the sensitivity of leukaemic cells to apoptotic stimuli.

Polyphenols represent a unique class of phytochemicals that possess excellent antioxidant, and antiinflammatory properties, and modulate cell signalling pathways leading to anti-cancer effects [21-23]. Epidemiological data has shown that diets rich in polyphenols significantly improve the quality of life and survival rates of patients with a range of chronic diseases, including cancer [21-24]. Recently, Montané et al., 2020 reported that using polyphenols, as an alternative to anti-cancer drugs is a very promising approach, because they minimize or suppress the adverse effects of standard therapies. One such polyphenol is apigenin, it is found at high levels in herbs, fruits, and vegetables, such as oregano, parsley, celery, celeriac, carrots, and chamomile tea $[23,24]$.

At present, there is evidence suggesting that apigenin has an anti-cancer effect on 14 different cancers including breast [25-30]; prostate [31-33]; colon [34-38]; bladder [39, 40]; cervical [41, 42]; lung [43]; oesophageal [44]; liver [45]; pancreatic [46]; and bone cancer [47]; as well as glioblastoma [48]; melanoma [49]; lymphoma [50]; and leukaemia [51-55]. Within leukaemia cell lines, apigenin suppresses cell proliferation, induces cell cycle arrest, and apoptosis of leukaemia cells, in vitro [55]. Interestingly, apigenin has no anti-proliferative effect in normal cells, suggesting its potential as an anti-cancer compound, with no toxicity to normal cells $[53,55]$. Hussain et al., 2010 also reported that apigenin has low intrinsic toxicity and limited mutagenic properties, compared to other structurally related flavonoids [50].

Most of these studies suggest that apigenin can induce apoptosis in cancers mainly via a mitochondrialdependent mechanism [26, 49-54]. Apigenin mediated downregulation of BCL-2, causes the release of cytochrome $c$ from the mitochondria to the cytosol, which leads to the activation of caspase- 9 and caspase-3 [26, 49-54]. Likewise, previous research has shown that apigenin induces caspase 3 activity and apoptosis in various types of lymphoid (Jurkat, CCRF-CEM, MOLT-3, U937) and myeloid (HL60, THP- 
$1, \mathrm{~K} 562, \mathrm{KG1a}$ ) leukaemia cell lines with minimum effect on non-tumour blood progenitor cells (CD34 ${ }^{+}$) [55]. Notably, apigenin produced a greater toxicity on lymphoid than myeloid leukaemia cells [55].

Furthermore, the use of combination treatments can improve the response to chemotherapy, clinical outcome, and patient survival rates [22, 56]. Montané et al., 2020 suggested that chemotherapy agents could be combined with polyphenols, as polyphenols could enhance the efficacy of chemotherapy agents and decrease the development of drug resistance, toxicity, and side effects on human health [23]. There have been promising results in in vitro and in vivo studies using combination treatments of apigenin and chemotherapeutic agents, this has opened a new avenue of drug discovery for cancer. Recent studies showed that apigenin can synergistically enhance the anti-cancer activity of 5-fluorouracil in the treatment of breast cancer cell lines (MDA-MB-453) [57] and head and neck cancer cell lines (SCC25 and A431) [58]; paclitaxel in the treatment of cervical cancer cell lines (HeLa) [59]; ABT-263 in the treatment of colon cancer cell lines (HTC-116) [60]; and cisplatin in the treatment of lung cancer cell lines (A549) [61]. In a recent study by Huang et al., 2020, they demonstrated apigenin synergistically enhanced the action of the BTK inhibitor abivertinib, and this action was associated with an apigenin-mediated downregulation of BCLX by the suppression of the PI3K/p-AKT pathway in diffuse large B-cell lymphoma cell lines (U2932 and OCl-LY10) at $24 \mathrm{~h}$ [62]. All these previous studies suggest that apigenin has great potential to improve cancer therapy.

This is supported by our previous work, which demonstrated apigenin could synergistically enhance the action of topoisomerase inhibitor agents (e.g., etoposide), and alkylating agents (e.g., cyclophosphamide) reducing ATP levels, and inducing apoptosis in both lymphoid and myeloid leukaemia cell lines; whilst protecting normal hematopoietic stem cells (CD133 ${ }^{+} \mathrm{HSC}$ and CD34 $\left.{ }^{+} \mathrm{HSC}\right)[63,64]$. However, the exact molecular mechanisms underlying proapoptotic effects associated with apigenin in combination with these chemotherapies have not been fully elucidated. The expression of genes that regulate apoptotic cell death plays an essential role in determining the sensitivity of cancer cells to chemotherapy [65]. For this reason, this study aims to analyse the expression profiles of intrinsic and extrinsic apoptosis-related genes and proteins, to help elucidate the molecular mechanisms of action of apigenin in combination with chemotherapy agents (etoposide and cyclophosphamide) on signaling molecules that play important roles in mechanisms in programmed cell death of acute lymphoid and myeloid leukaemia.

\section{Materials And Methods}

\subsection{Experimental design}

\subsubsection{Leukaemia cell lines}

Two human leukaemia cell lines were selected for this study: one acute myeloid leukaemia cell line [THP1 (monocytic leukaemia)] (ATCC: TIB- 202, Middlesex, UK) and one acute lymphoid leukaemia cell line [Jurkat (peripheral blood T cell leukaemia)] (ATCC: TIB-152, Middlesex, UK); these cell lines were treated with apigenin (AP) alone and in combination with two chemotherapy agents etoposide (ETP) and 
cyclophosphamide (CYCLO), which have been previously shown to act synergistically with apigenin [63, 64].

\subsubsection{Culture conditions}

Cells were cultured as described in Mahbub et al., 2013 [55], and incubated under standard cell culture conditions at $37^{\circ} \mathrm{C}$ with a $5 \% \mathrm{CO}_{2}$ atmosphere. Furthermore, cells were tested for mycoplasma contamination using the MycoAlert ${ }^{\mathrm{TM}}$ mycoplasma detection kit (Lonza, MD, USA) and were negative throughout all experiments.

\subsubsection{Treatment regimes}

Cells were seeded at $1 \times 10^{6}$ per well in 12-well plates and treated with the lowest significant doses (LSDs) of apigenin (AP) at $10 \mu \mathrm{M}$ and $50 \mu \mathrm{M}$, etoposide (ETP) at $0.01 \mu \mathrm{M}$ and cyclophosphamide (CYCLO) at $2 \mu \mathrm{M}$ and $10 \mu \mathrm{M}$, alone or in combination; along with a vehicle control for $24 \mathrm{~h}$. These LSDs were selected based on our earlier published data $[55,63,64]$ and caused a $10-20 \%$ increases in caspase-3 activity and induced apoptosis in THP-1 and Jurkat cells, when compared to the vehicle control. The therapeutic agents: AP, ETP, and CYCLO were prepared as described previously $[55,63,64]$.

Following treatments of THP-1 and Jurkat cells at $24 \mathrm{~h}$ with apigenin alone or in combination with etoposide and cyclophosphamide, an investigation was made of the expression of fourteen apoptosisrelated genes: BAD, BAX, BCL2, BCLX, CYT c, SMAC/DIABLO, HTRA2/OMI, TRAILR1/DR4, TNFR1/TNFRSF1A, FAS/CD95, FADD, CASP-8, CASP-9, and CASP-3; using a TaqMan ${ }^{8} H u m a n$ Apoptosis Array (Thermo Fisher Scientific-Applied Biosystems) based on the RT-qPCR reaction. This array is in a 96 well format. Each well contained an assay for the human apoptotic genes of interest plus four endogenous control genes: transferrin receptor (TFRC), glyceraldehyde-3-phosphate dehydrogenase (GAPDH), hypoxanthine phosphoribosyltransferase 1 (HPRT1), and Beta glucuronidase (GUSB) (Thermo Fisher Scientific-Applied Biosystems). This was performed once in three technical experiments for each treatment in THP-1 and Jurkat cell lines. This was performed in triplicate in three technical repeats for each treatment in THP-1 and Jurkat cell lines.

Subsequently, apigenin alone or in combination with etoposide and cyclophosphamide were also analysed for the expression of twelve apoptosis-related proteins: BAD, BAX, BCL2, BCLX, CYT $C$, SMAC/DIABLO, HTRA2/OMI, TRAILR1/DR4, TNFR1/TNFRSF1A, FAS/D95, FADD, and CASP-3; using the Proteome Profiler ${ }^{\mathrm{TM}}$ Array- Human Apoptosis Array Kit (R\&D Systems). In brief, the Human Apoptosis Proteome Profiler ${ }^{\text {TM }}$ array (R\&D Systems) was composed of a nitrocellulose membrane with duplicate spots for each apoptosis-related protein. Protein expression analysis was performed in duplicate on arrays (each array contained two spots for each protein analysed) for duplicate technical repeats for each treatment in each type of cell, thus a total of 4 repeats per protein per treatment group. All analyses were performed according to the manufacturer's instructions.

\subsection{Gene expression analysis using a TaqMan® Human Apoptosis Array based on the RT-qPCR reaction.}


Treated cells were collected and the RNA was extracted using an Ambion® PureLink ${ }^{\text {TM }}$ RNA Mini Kit (Thermo Fisher Scientific-Invitrogen) following the manufacturer's instructions. The purified RNA was then transcribed to cDNA using High-Capacity RNA-to-cDNA ${ }^{\mathrm{TM}}$ Kit (Thermo Fisher Scientific-Applied Biosystems) according to the manufacturer's instructions.

Following preparation of the cDNA, a $10 \mu$ of PCR reaction mix was prepared with the $5 \mu \mathrm{l}$ of cDNA and 5 $\mu$ of Taqman Fast Advanced Master Mix (Thermo Fisher Scientific- Applied Biosystems) following the manufacturer's instructions and loaded onto the TaqMan Array Human Apoptosis Fast 96-Well Plate (Thermo Fisher Scientific-Applied Biosystems). Next, the plate was loaded into the StepOnePlus ${ }^{\mathrm{TM}}$ Real- $^{-}$ Time PCR system (Applied Biosystems, Sheffield Hallam University, UK). Thermal cycling conditions consisted of a hold step for 20 seconds at $95^{\circ} \mathrm{C}$, and then amplification for 40 cycles starting with an initial denaturation step at $95^{\circ} \mathrm{C}$ for 3 seconds and an annealing/extending step at $60^{\circ} \mathrm{C}$ for 30 seconds. The relative gene expression was determined using the $2^{-\Delta \Delta C T}$ method [66]. The results are expressed as medians and ranges, of three independent repeats.

\subsection{Protein expression analysis using a proteome profiler $^{\mathrm{TM}}$ - human apoptosis array.}

Treated cells were collected and washed twice with PBS. Cells were lysed with CelLytic M (Sigma, C2978, $10 \mathrm{ml} / \mathrm{g}$ ) supplemented with a protease inhibitor cocktail (Sigma, P2714, 1:100) as described in the manufacturer's instructions. The total protein content was quantified using the Pierce ${ }^{\mathrm{TM}}$ BCA protein assay kit (Thermofisher Scientific), following the manufacturer's instructions. In brief, each antibodycoated profiler membrane (R\&D Systems) was incubated with $300 \mu \mathrm{g}$ of cell lysate with the ready-to-use pre-coated array membranes overnight at $4^{\circ} \mathrm{C}$ on a rocking platform.

Cell lysates were analysed using the proteome profiler human apoptosis antibody array kit (R\&D Systems) in accordance with the manufacturer's instructions. Thereafter, the array membranes were scanned using the LI-COR Odyssey ${ }^{\mathrm{R}}$ Infrared Imaging System (LI-COR). The pixel density of each duplicated array spot was quantified using Image Studio ${ }^{\mathrm{TM}}$ Software version 5.2 (LI-COR). Following the manufacturer's instructions ( $R \& D$ Systems), the mean pixel density of each duplicated protein spot was determined and subtracted from the mean density of the background/reference spots. Reference spots are included in each array to align the transparency overlay template and to demonstrate that the array has been incubated with IRDye ${ }^{\circledR} 800 \mathrm{CW}$ Streptavidin during the assay procedure. The results were expressed as a median and range of two independent experiments.

\subsection{Statistical analysis}

The median and range was determined for each assay. Stats Direct software (Stats Direct Ltd, Altrincham, UK) was used to determine if the data followed a normal distribution using a Shapiro-Wilk test. As data was identified as non-parametric, a Kruskal-Wallis with a Conover-Inman post-hoc test was used to determine statistical significance. Significance was set at $P \leq 0.05$. Statistical significance of individual agents was determined firstly in comparison to the vehicle control (VC). The statistical 
significance of combined apigenin and chemotherapy treatments was determined in comparison to the vehicle control and the individual treatments alone. The results were shown as relative fold change in gene expression/protein levels, with the vehicle control being set at 1 .

\subsubsection{Individual treatment effects}

A significant increase or decrease following individual treatments on apoptotic gene and protein expression was determined when compared to the vehicle control $(\mathrm{VC})(\mathrm{P} \leq 0.05)\left({ }^{*}\right)$. A significant increase is shown in Table 1 as an upward arrow in green ( $\uparrow$ ), whilst a significant decrease is shown as a downward arrow in red $(\downarrow)$. Where there were no significant changes in gene/protein expression compared to the vehicle control (VC); this was represented by a dash (-).

\subsubsection{Combination treatments effects:}

The effect of combination treatments on apoptotic gene and protein expression were classified either as:

- Synergistic - here there is a significant increase or decrease in gene/protein expression compared to the vehicle control $(\mathrm{VC})$ and drugs alone $(\mathrm{P} \leq 0.05)\left({ }^{*}\right)$. Synergistic increases in gene/protein expression are represented in the bar charts in dark green, whilst a synergistic decrease is represented in the bar charts in dark red.

- Non-interactive, but with significant effect - here there is a significant increase or decrease in gene/protein expression compared to the vehicle control (VC) and/or one of the individual drugs alone $(P \leq 0.05)(*)$. The significant increase in gene/protein expression is represented in the bar charts in light green, whilst a significant decrease is represented in the bar charts in pink.

- No effect - here there is no significant difference in gene/protein expression compared to the vehicle control (VC) and/or one of the individual drugs. This effect is represented in the bar charts in grey.

\section{Results}

\subsection{Effect of individual drug alone on apoptosis-related gene and protein expression in acute leukaemia cells:}

\subsubsection{Apigenin}

In THP-1 myeloid leukaemia cells, the use of the LSD for apigenin of $10 \mu \mathrm{M}$, caused a significant increase of all pro-apoptosis-related genes and proteins (BAD, BAX, CYT $c$, HTRA2/OMI, SMAC/DIABLO, TRAILR1/DR4, TNFR1/TNFRSF1A, FAS/CD95, FADD, CASP-8, CASP-9 and CASP-3), when compared to the vehicle control $(P \leq 0.05)$. Whilst anti-apoptotic genes and proteins (BCL2 and BCLX) were unchanged (Table 1).

In contrast, Jurkat lymphoid leukaemia cells when treated with the LSD for apigenin ( $50 \mu \mathrm{M})$ for $24 \mathrm{~h}$, there was a significant increase in nine pro-apoptotic genes and/or proteins (BAD, BAX, SMAC/DIABLO, 
TRAILR1/DR4, TNFR1/TNFRSF1A, FAS/CD95, CASP-8, CASP-9 and CASP-3) compared to the vehicle control $(P \leq 0.05)$, with no changes seen in the remaining investigated genes and proteins (CYT $C$, HTRA2/OMI, FADD, BCL2, and BCLX) (Table 1).

\subsubsection{Etoposide}

Treatment of THP-1 and Jurkat cells with the LSD of etoposide $(0.01 \mu \mathrm{M})$ for $24 \mathrm{~h}$ significantly increased the expression of the pro-apoptotic genes: TNFR1/TNFRSF1A, CASP-9, and CASP-3 compared to the vehicle control $(P \leq 0.05)$ (Table 1), plus there was an increase of BAX expression in the Jurkat lymphoid leukaemia cells when compared to the vehicle control $(P \leq 0.05)$ (Table 1$)$.

Etoposide treatment also caused a significant decrease of the anti-apoptotic factor BCL2 gene expression and protein levels in THP-1 myeloid leukaemia cells, when compared to the vehicle control $(P \leq 0.05)$ (Table 1). However, there was no significant effect on the remaining investigated apoptotic genes or proteins in both cell lines (Table 1).

\subsubsection{Cyclophosphamide}

Following treatment with the LSD of cyclophosphamide for THP-1 myeloid $(2 \mu \mathrm{M})$ and Jurkat lymphoid $(10 \mu \mathrm{M})$ leukaemia cells for $24 \mathrm{~h}$, this caused differential effects on the apoptosis genes and proteins (Table 1). In THP-1 acute myeloid leukaemia cells, there was a significant decrease in the expression of BCL2, BCLX, and BAD genes and proteins; with a significant increase in TNFR1/TNFRSF1A, CASP-9, and CASP-3 gene expression, when compared to the vehicle control $(P \leq 0.05)$ (Table 1).

In Jurkat acute lymphoid leukaemia cells, there was a significant increase of the expression of BAD, BAX, and CYT $c$ genes and proteins, as well as CASP-9 and CASP-3 genes when compared to the vehicle control $(P \leq 0.05)$ (Table 1). However, there were no significant effects on the remaining apoptotic genes or proteins in either cell line (Table 1). 
Table 1

A summary of the effects of individual treatments: apigenin (AP), etoposide (ETP), and cyclophosphamide (CYCLO) on the expression of apoptosis-related genes and proteins in acute myeloid (THP-1) and lymphoid (Jurkat) leukaemia cell lines; using their lowest significant doses (LSDs) that induce apoptosis (previously determined in Mahbub et al., 2013; 2015; 2019) for $24 \mathrm{~h}$ treatment. A Kruskal Wallis with an Inman post-hoc test was used to determine statistical significance and $P \leq 0.05$. The individual treatment effects were determined as described in Section 2.4.

Effect of Treatments Alone on Acute Leukaemia Cells using their LSDs at $24 \mathrm{~h}$

\begin{tabular}{|c|c|c|c|c|c|c|c|}
\hline \multirow[t]{3}{*}{$\begin{array}{l}\text { Name of Apoptosis } \\
\text { Pathways }\end{array}$} & \multirow{2}{*}{$\begin{array}{l}\text { Apoptotic } \\
\text { Genes and Proteins }\end{array}$} & \multicolumn{3}{|c|}{$\begin{array}{l}\text { THP-1 Acute Myeloid } \\
\text { Cells }\end{array}$} & \multicolumn{3}{|c|}{$\begin{array}{l}\text { Jurkat Acute } \\
\text { Lymphoid Cells }\end{array}$} \\
\hline & & AP & ETP & CYCLO & AP & ETP & CYCLO \\
\hline & & $\begin{array}{l}10 \\
\mu \mathrm{M}\end{array}$ & $\begin{array}{l}0.01 \\
\mu M\end{array}$ & $2 \mu \mathrm{M}$ & $\begin{array}{l}50 \\
\mu \mathrm{M}\end{array}$ & $\begin{array}{l}0.01 \\
\mu \mathrm{M}\end{array}$ & $10 \mu \mathrm{M}$ \\
\hline
\end{tabular}

\begin{tabular}{|c|c|c|c|c|c|c|c|c|}
\hline \multirow[t]{4}{*}{ BCL2 Antiapoptotic } & \multirow[t]{2}{*}{ BCL2 } & Gene & - & $\downarrow$ & $\downarrow$ & - & - & - \\
\hline & & Protein & - & $\downarrow$ & $\downarrow$ & - & - & - \\
\hline & \multirow[t]{2}{*}{ BCLX } & Gene & - & - & $\downarrow$ & - & - & - \\
\hline & & Protein & - & - & $\downarrow$ & - & - & - \\
\hline \multirow[t]{4}{*}{ BCL2 Proapoptotic } & \multirow[t]{2}{*}{ BAD } & Gene & $\uparrow$ & - & $\downarrow$ & $\uparrow$ & - & $\uparrow$ \\
\hline & & Protein & $\uparrow$ & - & $\downarrow$ & $\uparrow$ & - & $\uparrow$ \\
\hline & \multirow[t]{2}{*}{ BAX } & Gene & $\uparrow$ & - & - & $\uparrow$ & $\uparrow$ & $\uparrow$ \\
\hline & & Protein & $\uparrow$ & - & - & $\uparrow$ & & $\uparrow$ \\
\hline \multirow{6}{*}{$\begin{array}{l}\text { Mitochondrial } \\
\text { Members }\end{array}$} & \multirow[t]{2}{*}{ CYT c } & Gene & $\uparrow$ & - & - & - & - & $\uparrow$ \\
\hline & & Protein & $\uparrow$ & - & - & - & - & $\uparrow$ \\
\hline & \multirow[t]{2}{*}{ HTRA2/OMI } & Gene & $\uparrow$ & - & - & - & - & - \\
\hline & & Protein & $\uparrow$ & - & - & - & - & - \\
\hline & \multirow[t]{2}{*}{ SMAC/DIABLO } & Gene & $\uparrow$ & - & - & $\uparrow$ & - & - \\
\hline & & Protein & $\uparrow$ & - & - & $\uparrow$ & - & - \\
\hline \multirow{6}{*}{$\begin{array}{l}\text { Death Receptors \& } \\
\text { Domain }\end{array}$} & \multirow[t]{2}{*}{ TRAILR1/DR4 } & Gene & $\uparrow$ & - & - & $\uparrow$ & - & - \\
\hline & & Protein & $\uparrow$ & - & - & $\uparrow$ & - & - \\
\hline & \multirow{2}{*}{$\begin{array}{l}\text { TNFR1/ } \\
\text { TNFRSF1A }\end{array}$} & Gene & $\uparrow$ & $\uparrow$ & $\uparrow$ & $\uparrow$ & $\uparrow$ & - \\
\hline & & Protein & $\uparrow$ & - & - & $\uparrow$ & - & - \\
\hline & \multirow[t]{2}{*}{ FAS/CD95 } & Gene & $\uparrow$ & - & - & $\uparrow$ & - & - \\
\hline & & Protein & $\uparrow$ & - & - & $\uparrow$ & - & - \\
\hline
\end{tabular}


Effect of Treatments Alone on Acute Leukaemia Cells using their LSDs at $24 \mathrm{~h}$

\begin{tabular}{|lllllllll}
\hline \multirow{4}{*}{ Caspases } & FADD & Gene & $\uparrow$ & - & - & - & - & - \\
\cline { 3 - 8 } & & Protein & $\uparrow$ & - & - & - & - & - \\
& CASP-8 & Gene & $\uparrow$ & - & - & $\uparrow$ & - & - \\
\cline { 2 - 8 } & CASP-9 & Gene & $\uparrow$ & $\uparrow$ & $\uparrow$ & $\uparrow$ & $\uparrow$ & $\uparrow$ \\
\cline { 3 - 7 } & CASP-3 & Gene & $\uparrow$ & $\uparrow$ & $\uparrow$ & $\uparrow$ & $\uparrow$ & $\uparrow$ \\
& & Protein & $\uparrow$ & - & - & $\uparrow$ & - & - \\
\hline
\end{tabular}

\subsection{Effect of combination treatments on expression of BCL-2 family anti-apoptotic genes and proteins (BCL2 and BCLX)}

When apigenin was used in combination with etoposide, there was a synergistic decrease of BCL2 gene expression in both myeloid (THP-1) and lymphoid (Jurkat) leukaemia cells (Figure 1A). Similarly, when apigenin was used in combination with cyclophosphamide there was a synergistic decrease on BCL2 gene expression in Jurkat lymphoid leukaemia cells only $(P \leq 0.05)$ (Figure $1 A)$, when compared to the vehicle control and drugs alone, $(P \leq 0.05)$ (Figure $1 A)$. Importantly, however the protein expression for BCL2, was unchanged during apigenin and etoposide combination treatments, in both THP-1 and Jurkat cells (Figure 1B). There was however a significant decrease when compared to the vehicle control, and/or one of the treatments alone $(P \leq 0.05)$ (Figure 1B).

Apigenin when used in combination with etoposide was shown to synergistically decrease the expression of BCLX gene and protein levels in Jurkat lymphoid leukaemia cells, compared to the vehicle control and drugs alone $(P \leq 0.05)$ (Figure $1 A)$. Similarly, apigenin when used in combination with cyclophosphamide, also caused a significant decrease in the expression of BCLX gene and protein in Jurkat cells (Figure 1A \& B) when compared to the vehicle control and/or one of the individual treatments $(P \leq 0.05)$. In contrast, in THP-1 myeloid cells, apigenin when combined with cyclophosphamide and etoposide caused a significant reduction in BCLX gene expression (Figure 1A), whilst protein levels remained unchanged (Figure 1B).

Overall, Jurkat lymphoid cells were more susceptible to combination treatments, which caused a reduction in anti-apoptotic genes and proteins; whilst the THP-1 myeloid cells were unresponsive (Figure $1 \mathrm{~A} \& \mathrm{~B}$ ). Examples of the apoptosis-related proteins (BCL2 and BCLX) array for THP-1 (Supplementary Figure 1) and Jurkat (Supplementary Figure 2) cells are included.

\subsection{Effect of combination treatments on expression of BCL-2 family pro-apoptotic genes and proteins (BAD and $B A X)$}

There were no effects on the expression of BAD gene and protein in the THP-1 myeloid leukaemia cells when treated with each of the combination regimes when compared to the vehicle controls (Figure 2B). 
However, when Jurkat lymphoid leukaemia cells were treated with a combination of apigenin and cyclophosphamide there was a synergistic increase in the expression of BAD gene and protein levels compared to the vehicle controls and drugs alone, $(P \leq 0.05)$ (Figure $2 A \& B)$. However, when apigenin was combined with etoposide in Jurkat cells there was a significant increase in BAD gene expression when compared to the vehicle controls and/or one of the individual drugs, $(P \leq 0.05)$ (Figure 2A); but protein expression was unchanged (Figure $2 A \& B$ ). Interestingly, all investigated combination treatments caused a synergistic increase in the expression of BAX gene and protein levels in both acute myeloid and lymphoid leukaemia cells (THP-1 and Jurkat) when compared to the vehicle controls and drugs alone, ( $P$ $\leq 0.05$ ) (Figure 2A \& B). Examples of the apoptosis-related proteins (BAX and BAD) array for THP-1 (Supplementary Figure 1) and Jurkat (Supplementary Figure 2) cells are included.

\subsection{Effect of combination treatments on expression of mitochondrial genes and proteins (CYT c, SMAC/DIABLO, HTRA2/OMI)}

In both acute myeloid and lymphoid leukaemia cells (THP-1 and Jurkat), all the investigated combination treatments synergistically increased the gene and protein expression of CYT $c$ (Figure 3A \& B) and SMAC/DIABLO (Figure 4A \& B), when compared to the vehicle controls and drugs alone, $(P \leq 0.05)$. Similarly, there was a synergistic increase in the expression of HTRA2/OMI gene and protein in Jurkat lymphoid leukaemia cells when treated with all the combination treatments $(P \leq 0.05)$ (Figure 4A \& B). However, in THP-1 myeloid leukaemia cells, there was a significant increase in the expression of HTRA2/OMI gene and protein when compared to the vehicle controls and/or one of the individual drugs $(P \leq 0.05)$ (Figure 4A \& B). Examples of the apoptosis-related proteins (CYT C, SMAC/DIABLO, HTRA2/OMI) array for THP-1 (Supplementary Figure 1) and Jurkat (Supplementary Figure 2) cells are included.

\subsection{Effect of combination treatments on expression of death receptors/domain genes and proteins (TRAILR1/DR4, TNFR1/TNFRSF1A, FAS/CD95, FADD)}

In THP-1 acute myeloid cells, the combination treatment of apigenin and etoposide caused a synergistic increase in TNFR1/TNFRSF1A gene and protein expression, when compared to the vehicle controls and drugs alone $(P \leq 0.05)$ (Figure $5 A \& B)$. Whilst, when apigenin was used in combination with cyclophosphamide, it caused a significant increase in the expression of TNFR1/TNFRSF1A gene compared to the vehicle controls and/or one of the individual drugs, $(P \leq 0.05)$ (Figure $5 A)$; although protein expression was unchanged (Figure 5B). Therefore, in THP-1 cells, both combination treatments caused a significant increase in gene and protein expression of TRAILR1/DR4 (Figure 5), and gene expression in FAS/CD95 and FADD, when compared to the vehicle control and/or one of the individual drugs, $(P \leq 0.05)$; with differential effects being seen in protein expression of FAS/CD95, and FADD (Figure 6). 
In Jurkat acute lymphoid cells, the combination treatment of apigenin and etoposide caused a significant increase in the expression of all investigated death receptors and domain genes and proteins when compared to the vehicle controls and/or one of the individual drugs, ( $\leq \leq 0.05)$ (Figure $5 \& 6)$. In contrast, the combination treatment of apigenin and cyclophosphamide, caused a synergistic increase in the expression of TRAILR1/DR4 gene and protein levels, when compared to the vehicle controls and drugs alone $(P \leq 0.05)$ (Figure $5 A \& B)$. This combination did not cause any significant effect on the expression of TNFR1/TNFRSF1A (Figure 5A \& B) FAS/CD95, and FADD (Figure 6A \& B) genes and proteins.

Examples of the apoptosis-related proteins (TRAILR1/DR4, TNFR1/TNFRSF1A, FAS/CD95, FADD) array for THP-1 (Supplementary Figure 1) and Jurkat (Supplementary Figure 2) cells are included.

\subsection{Effect of combination treatments on expression of caspase genes and proteins (CASP-8, CASP-9, CASP-3)}

In both myeloid and lymphoid leukaemia cell lines, all the combination treatments caused a synergistic increase on CASP-9 and CASP-3 gene expression when compared to the vehicle control and drugs alone $(P \leq 0.05)$ (Figure 7A) and CASP-8 when compared to the vehicle control and/or one of the individual drugs $(P \leq 0.05)$ (Figure 7A). The CASP-3 protein expression was significantly increased in both THP-1 and Jurkat cells when compared to the vehicle control and/or one of the individual drugs $(P \leq 0.05)$ (Figure 7B) (Supplementary Figure 1 \& 2).

\section{Discussion}

Our earlier work has shown that polyphenols can decrease cell proliferation, cause cell cycle arrest, and induce apoptosis in leukaemia cell lines $[23,55]$. One polyphenol which has been shown to have potential at inducing apoptotic cell death in leukaemia cells is apigenin $[23,55]$. Work reported by ourselves and others has shown that apigenin can inhibit cell proliferation, and induce apoptosis in a number of myeloid (HL60 (human promyelocytic leukaemia) [51, 55, 67], THP-1 (acute monocytic leukaemia) [53, 55], KG-1a (acute myelogenous leukaemia), K562 (chronic myeloid leukaemia)), and lymphoid (Jurkat (peripheral blood T cell lymphoid leukaemia), MOLT-3 (acute lymphoblastic leukaemia patient relapsed following chemotherapy), CCRF-CEM (acute lymphoblastic leukaemia) [55], and U937 (histiocytic lymphoma) $[54,55]$ leukaemia cell lines; with minimum effect on non-tumour control haematopoietic stem cells $\left(\mathrm{CD} 34^{+}\right)$[55]. The observed differential effect of apigenin on leukaemia and non-tumour haematopoietic stem cells suggests that apigenin may have therapeutic potential in the treatment of leukaemia [55].

Previously, we have shown that apigenin acts synergistically with chemotherapy agents such as etoposide (a topoisomerase inhibitor) and cyclophosphamide (an alkylating agent), causing a reduction in ATP and glutathione levels, DNA damage and induction of apoptosis in acute myeloid (THP-1) and lymphoid (Jurkat) leukaemia cell lines $[63,64]$. To progress our understanding of the therapeutic potential of apigenin, it is essential to elucidate their molecular mechanism of action during apoptosis. Here we 
investigated the molecular events that trigger apoptosis in response to apigenin alone, and in combination with etoposide and cyclophosphamide in both acute myeloid (THP-1) and lymphoid (Jurkat) leukaemia cells, following treatment for $24 \mathrm{~h}$. We measured eight key genes/proteins of the intrinsic apoptosis pathway (BAD, BAX, BCL2, BCLX, CYT c, SMAC/DIABLO, HTRA2/OMI, CASP-9); and five genes/proteins of the extrinsic pathway (TRAILR1/DR4, TNFR1/TNFRSF1A, FAS, FADD, CASP-8); plus, the execution caspase 3 (CASP-3). There are two core pathways involved in inducing apoptosis: (1) The intrinsic or mitochondrial-mediated pathway, and (2) the extrinsic or death receptor-mediated pathway [5, 16]. Initiation of either pathway ultimately results in a caspase activation cascade and cellular death [5, $16,17]$.

Here, apigenin when used alone in THP-1 and Jurkat cells caused no effect on the expression of antiapoptotic BCL2 and BCLX genes and proteins. However, apigenin did cause an increase in the gene expression and protein levels of pro-apoptotic $B A X$ and $B A D$ of the mitochondrial pathway. This was associated with an increase in the expression of SMAC/DIABLO and CASP-9 genes and proteins, in both acute leukaemia (THP-1 and Jurkat) cells. At the same time, apigenin also targeted the apoptosis death receptor pathway by increasing the gene and protein expression of TRAILR1/DR4, TNFR1/TNFRSF1A, and FAS/CD95; which then increased the gene expression of CASP-8, in both acute leukaemia cell lines. This subsequently led to an increase in the expression of CASP-3 gene and protein levels. This is consistent with previous work, where apigenin, induced apoptosis in leukaemia cells (HL60, THP-1, U937) via mitochondrial-dependent mechanisms, with the release of cytochrome $c$ to the cytosol and the activation of caspase-9 and -3 [51-54]. However, it is important to note that Wang et al., 1999 reported that apigenin could also induce apoptosis via an extrinsic pathway through the activation of caspase 8 , in HL60 cells [51].

Etoposide and cyclophosphamide alone were shown here to induce apoptosis, while their effect was different in each leukaemia cell line. In THP-1 myeloid cells, both etoposide and cyclophosphamide alone caused a decrease in expression of anti-apoptotic BCL2 and/or BCLX, and an increase in the expression of TNFR1/TNFRSF1A. This was associated with the increase in the expression of CASP-9 and -3 genes. In Jurkat lymphoid cells, etoposide alone increased the expression of BAX, TNFR1/TNFRSF1A, CASP-9, and -3 genes. Whereas cyclophosphamide was much more effective in Jurkat cells and seemed to specifically target intrinsic apoptosis; and increased the gene and protein expression of pro-apoptotic BAD and BAX, CYT C, CASP-9 and -3. It was clear that etoposide and cyclophosphamide alone increased the expression of CASP-9 and -3 in both acute leukaemia cell lines. Studies reported that most chemotherapeutic drugs induce mitochondrial membrane permeabilization prior to apoptosis $[65,68]$.

This study has shown that exposure of acute leukaemia cells whether myeloid (THP-1) or lymphoid (Jurkat) cells, to combination treatments of apigenin and chemotherapy agents (etoposide or cyclophosphamide), induced apoptosis mainly through the intrinsic apoptotic pathway. With the major effect being, that apigenin enhances the pro-apoptotic activity of chemotherapy agents, through the synergistic increase in expression of BAX, CYT c, SMAC/DIABLO, HTRA2/OMI, CASP-9, and -3 gene and protein levels, whilst decreasing anti-apoptotic BCL2 gene expression (Figure 8 and 9). BCL-2 family 
members are known to play a critical role in regulating changes in mitochondrial outer membrane permeability and apoptosis induction [65]. Normally, downregulation of anti-apoptotic BCL-2 (as BCL2 or $B C L X$ ) and upregulation of pro-apoptotic BCL-2 family proteins (such as BAX) are associated with the mechanism of cell death [16]. BAX allows the release of cytochrome $c$ (CYT c) from the mitochondrial intermembrane space into the cytosol to promote the induction of apoptosis [16].

Hassanpour et al., 2018 reported that the dysfunction of the intrinsic apoptotic pathway is a key strategy to avoid apoptosis in leukaemia cells [2]. Acute leukaemia is commonly associated with a decrease in cell sensitivity to pro-apoptotic signals due to overexpression of anti-apoptotic proteins of the BCL-2 family such as BCL2 and BCLX [3, 9, 10, 16, 69]; and/or with low levels of pro-apoptotic members such as BAX [11]. Yoo et al., 2012 and Jan et al., 2019 reported that targeting the expression of anti-apoptotic and/or pro-apoptotic members of the apoptotic pathways is a promising strategy to induce cancer cell death and improve sensitivity to chemotherapy agents $[16,65]$. Here, the effective targeting of anti-apoptotic members BCL2 and BCLX, by the use of apigenin with standard chemotherapy agents; seem to vary dependent on the type of leukaemia cell line and chemotherapy agent used. Apigenin showed a greater modulation of anti-apoptotic members in lymphoid, compared to myeloid cells. In Jurkat acute lymphoid leukaemia cells, when treated with apigenin in combination with either etoposide or cyclophosphamide, there was a synergistic decrease of anti-apoptotic BCL2 gene expression, which was accompanied by a decrease of BCLX, when treated with a combination of apigenin and etoposide. Similar reductions in BCLX expression in Jurkat cells have been seen when apigenin was used in combination with TRAIL [70].

In contrast here, THP-1 acute myeloid leukaemia cells, showed less modulation by the apigenin combination therapies. But the use of apigenin with etoposide, did however cause a synergistic decrease of BCL2 expression. Cassier et al., 2017 reported that downstream effectors of the mitochondrial pathway are mainly deregulated in acute myeloid leukaemia (AML) through overexpression of antiapoptotic proteins of BCL2 and BCLX [69]. Indeed, BCL2 was the first apoptosis-related gene identified to play a role in carcinogenesis [16]. Overexpression of anti-apoptotic proteins BCL2 and BCLX inhibit apoptosis; and are reported to be correlated with the pathogenesis of acute leukaemia (mainly AML), and can induced resistance to chemotherapeutics $[16,17,65]$.

Notably, both acute leukaemia cells (THP-1 and Jurkat) showed a synergistic increase in gene and protein expression of proapoptotic BAX when treated with all combination treatments (Figure 8 and 9), whilst in Jurkat cells when treated with apigenin and cyclophosphamide, this was accompanied by a synergistic increase of BAD (Figure 9). In a similar study, when apigenin was used in combination with the BCL2 inhibitor navitoclax for $48 \mathrm{~h}$, there was an upregulation of BAX and BIM gene expression and protein levels, which led to apoptotic cell death in colon cancer (HTC-116) cells [60]. Likewise, Chen et al., 2019 showed that apigenin could inhibit tumorigenesis and induce apoptosis in cisplatin-resistant colon cancer cells (HT-295), when grown in vitro and in vivo as mouse xenografts, through the upregulation of pro-apoptotic BAX and downregulation of anti-apoptotic BCL2 [71]. 
Conventional chemotherapy agents used clinically, are known to indirectly activate BAX [72]. Liu et al., 2016 and Walensky et al., 2019 reported that the direct activation of BAX, holds great promise for cancer therapies, with the advantages of specificity, and the potential of overcoming chemo- and radioresistance; and that this is a possible target for small-molecule drug discovery $[72,73]$. Here, the synergistic increase of BAX gene and protein expression in both acute leukaemia cells (THP-1 and Jurkat) with all combination treatments, was associated with a synergistic increase in CYT $c$ and SMAC/DIABLO, and/or HTRA2/OMl gene and protein expression (Figure 8 and 9).

Cytochrome $c(\mathrm{CYT} c)$ is considered to be a critical mediator and biomarker in mitochondria-mediated apoptosis [74]. It is located on the inner surface of the mitochondria, it is a heme-containing metalloprotein and multifunctional enzyme, that is involved in cell apoptosis [74]. Functionally, overexpression of cytochrome $c$ effectively suppresses the proliferation of cancer cells and induces cell apoptosis, whilst the knockdown of cytochrome c, reverses these effects [74]. SMAC/DIABLO and HTRA2/OMI are important also to neutralize the inhibitory effect of inhibitors of apoptosis proteins (IAPs) on caspase-9 and -3 [68]. So, the release of cytochrome $c$, SMAC/DIABLO, and HTRA2/OMI promote the activation of initiator caspase- 9 and subsequently the executioner caspases $(6,7$ and 3$)$, which leads to apoptotic cell death $[68,74]$.

Cytochrome $c$ is the major inducer of caspase activation downstream of the mitochondrial pathway [76]. Cytochrome $c$ and procaspase-9 form the core of the mitochondria-mediated intrinsic apoptosis pathway [76]. Here, the gene expression of caspase 9 was synergistically increased in both THP-1 and Jurkat cells when treated with combination treatments of apigenin and etoposide or cyclophosphamide (Figure 8 and 9), as a result of increased BAX, CYT $c$, and SMAC/DIABLO activation. Similarly, three previous studies found that apigenin could synergistically increase caspase 9 gene expression, when used in combination with: (1) TRAIL for $24 \mathrm{~h}$ in Jurkat acute lymphoblastic leukaemia cells [70], (2) Abivertinib for $24 \mathrm{~h}$ in U2932 and OVI-LY1 diffuse large B-cell lymphoma cells; via the downregulation of PI3K/mTOR [62]; and (3) Cisplatin in A549 lung cancer cells [61]. In addition, Horinka et al., 2006 and Huang et al., 2020 also reported that apigenin/chemotherapy combination treatments synergistically upregulated caspase 8 activity in Jurkat, U2932, and OCI-LY1 cells $[62,70]$.

Here, we also showed that our apigenin/chemotherapy combination treatments mostly caused a significant increase or no effect on the expression of death receptors-related genes and proteins. The only synergistic increases were seen in TNFR1/TNFRSF1A in THP-1 myeloid leukaemia cells, when treated with apigenin and etoposide (Figure 8); and in TRAILR1/DR4 in Jurkat lymphoid leukaemia cells when treated with apigenin and cyclophosphamide (Figure 9). It is suggested that apigenin in combination with these chemotherapies, has less effect on the extrinsic compared to the intrinsic apoptosis pathway, as shown in Figure 8 and 9. There are also two earlier studies that found that apigenin can target the extrinsic pathway of apoptosis when used in combination with: (1) TRAIL in human acute lymphoblastic leukaemia cells (Jurkat) via upregulation of the expression of the DR5 gene [70]; and (2) 5-fluorouracil and cisplatin in head and neck carcinoma cells (SCC25 and A431) via upregulation of the expression of 
TNFR and TRAILR genes [58]. Thus here, the role of apigenin in the induction of the death receptor in acute leukaemia appears less clear [69].

Despite this, in this study, both THP-1 and Jurkat cells when treated with all apigenin/chemotherapy combination therapies did show an increase of caspase 8 activity, which promoted caspase 3 activation and apoptotic cell death. This is consistent, with findings in our earlier work in which a variety of combination treatments increased caspase 3 activity and induced apoptosis in THP- 1 and Jurkat cells $[63,64]$.

Interestingly, the current study showed that combination treatments synergistically increased caspase 3 activity via the mitochondrial pathways, as a consequence of increased BAX, CYT c, SMAC/Diablo, HTRA2/OMI and CASP-9 gene/protein expression, plus the reduction of BCL2 anti-apoptotic gene expression in both THP-1 and Jurkat cells (Figure 8 and 9). Previous work has also shown that the expression of caspase 3 can be synergistically increased when apigenin is used in combination with: (1) TRAIL at $24 \mathrm{~h}$ for acute lymphoblastic leukaemia cells (Jurkat) via upregulation of DR5 expression [70], (2) Abivertinib at $24 \mathrm{~h}$ for diffuse large B-cell lymphoma cells (U2932 and OCl-LY1) via downregulation of $\mathrm{PI3K} / \mathrm{mTOR}$ expression [62]; (3) 5-Fluorouracil at $72 \mathrm{~h}$ for breast cancer cells (MDA-MB-453) via downregulation of Akt expression [57]; and (4) Cisplatin for lung cancer cells (A549) [61].

Similarly, in our earlier work we showed that polyphenols, including apigenin could synergistically enhance the action of chemotherapeutic agents, including etoposide [63] and cyclophosphamide [64] in leukaemia cells (including Jurkat and THP-1), through a reduction of glutathione levels. Furthermore, these studies found that these polyphenol/chemotherapy combination treatments synergistically reduced cell ATP levels, arrested cell cycle, caused DNA damage, and induced apoptosis [63, 64]. Here, we have shown that the same combination treatments in the same cell lines targeted the mitochondrial pathway to induce apoptosis. Interestingly, many researchers reported that there is a strong correlation between glutathione depletion and restored apoptosis induction [75, 77]. Franco et al., 2009 reported that a depletion in the glutathione content can directly activate the intrinsic apoptotic pathway, either by: activating the initiator $\mathrm{BAX}$, releasing the cytochrome $\mathrm{c}$ from the mitochondria, or by forming the apoptosome; which can be oxidised for its pro-apoptotic action. This may explain why the investigated combination agents here, target the intrinsic apoptotic pathway and synergistically increased the expression of mitochondrial BAX and cytochrome $c$. Franco et al., 2009 reported that the depletion of glutathione, restored apoptosis via the intrinsic apoptotic pathway; and suggested that this could be a highly effective way to increase the efficacy of chemotherapy or anti-cancer agents $[75,77]$. In addition, Traverso et al., 2013 reported that high glutathione levels are commonly found in cancer cells and the efflux of glutathione is one of the major key mechanisms in the development of multi-drug resistance in cancer [77]. Our earlier work has found that the basal glutathione levels of the leukaemia cell lines were linked with the sensitivity to the treatments of polyphenol and chemotherapy [63]. It was demonstrated that Jurkat lymphoid leukaemia cell lines had lower basal glutathione levels than the THP-1 myeloid cell lines and non-tumour control cells $[63,64]$. This could explain why the Jurkat acute lymphoid cell lines 
are more sensitive and susceptible to the treatments with polyphenol and chemotherapy, either alone or in combination compared to the THP-1 acute myeloid cell lines.

Thus, this study has demonstrated the molecular events that trigger apoptosis in response to apigenin treatment, when used in combination with etoposide and cyclophosphamide in Jurkat and THP1 acute leukaemia cells. In conclusion, the combination of apigenin with these chemotherapy agents for treatment of acute leukaemia cells, has been shown to induce apoptosis by the increase of BAX expression (Figure 8 and 9), the permeabilization of the mitochondrial membrane, and the release of CYT c, SMAC/DIABLO, and HTRA2/OMI, which then promotes caspase-9 and -3 activation (Figure 8 and 9). It can be concluded that apigenin alone and/or in combination with standard chemotherapies induces apoptosis mainly through the activation of a mitochondrial-dependent pathway in lymphoid and myeloid leukaemia cells, and thus could have therapeutic potential in the treatment of leukaemia.

\section{Declarations}

Acknowledgments and Funding: This research did not receive any specific grant and it was self-funded by the major author from Faculty of Applied Medical Sciences - Umm Al Qura University, Saudi Arabia; and supported by the remaining authors and performed in Biomolecular Sciences Research Centre Sheffield Hallam University, United Kingdom.

Author contributions: All authors developed the program of research, took part in the analysis and interpretation of the data and the writing of the manuscript. The practical work was completed by Dr Amani A. Mahbub.

Competing interests: The authors declare no competing interests.

Additional Information: Supplementary information.

Correspondence and requests for materials should be addressed to Dr Nicola Jordan-Mahy.

\section{References}

1. International Agency for Research on Cancer. Available at https://gco.iarc.fr/today/fact-sheetscancers. (Accessed 01 June 2021).

2. Hassanpour, S. H., Dehghani, M. A., Karami, S. Z. \& Dehghani, F. Role of Apoptosis and Mitochondrial Dysfunction in the Treatment of Leukemia Types. Annal of Blood Cancer. 1, 1002 (2018).

3. McBride, A., Houtmann, S., Wilde, L., Vigil, C., Eischen, C. M., Kasner, M. \& Palmisiano, N. The Role of Inhibition of Apoptosis in Acute Leukemias and Myelodysplastic Syndrome. Frontiers in oncology. 9, 192 (2019).

4. Dong, Y., Shi, O., Zeng, Q., Lu, X., Wang, W., Li, Y. \& Wang, Q. Leukemia incidence trends at the global, regional, and national level between 1990 and 2017. Experimental hematology \& oncology. 9, 1-14 (2020). 
5. Vitale, I., Aaronson, S. A., Alnemri, E. S., Anews, D. W., Annicchiarico-Petruzzelli, M., Antonov, A. V. \& et al. Molecular mechanisms of cell death: recommendations of the Nomenclature Committee on Cell Death 2018. Cell death and differentiation. 25, 486-541 (2018).

6. Moriai, R., Asanuma, K., Kobayashi, D., Yajima, T., Yagihashi, A., Yamada, M. \& Watanabe, N. Quantitative analysis of the anti-apoptotic gene Survivin expression in malignant haematopoietic cells. Anticancer research. 21, 595-600 (2001).

7. Schuler, D. \& Szende, B. Apoptosis in acute leukemia. Leukemia Research. 28, 661-666 (2004).

8. Testa, U. \& Riccioni, R. Deregulation of apoptosis in acute myeloid leukemia. Haematologica. 92, 8194 (2007).

9. Konopleva, M., Contractor, R., Tsao, T., Samudio, I., Ruvolo, P. P., Kitada, S., Deng, X. \& et al. Mechanisms of apoptosis sensitivity and resistance to the $\mathrm{BH} 3$ mimetic ABT-737 in acute myeloid leukemia. Cancer cell. 10, 375-388 (2006).

10. Droin, N., Guéry, L., Benikhlef, N. \& Solary, E. Targeting apoptosis proteins in hematological malignancies. Cancer letters. 332, 325-334 (2011).

11. Kitada, S., Pedersen, I. M., Schimmer, A. D. \& Reed, J. C. Dysregulation of apoptosis genes in hematopoietic malignancies. Oncogene. 21, 3459-3474 (2002).

12. Onciu, M. Acute Lymphoblastic Leukemia. Hematol Oncol Clin N Am. 23, 655-674 (2009).

13. Terwilliger, T. \& Abdul-Hay, M. Acute lymphoblastic leukemia: a comprehensive review and 2017 update. Blood cancer journal. 7, 577 (2017).

14. Chen, B., Lee, J. B., Kang, H., Minden, M. D. \& Zhang, L. Targeting chemotherapy-resistant leukemia by combining DNT cellular therapy with conventional chemotherapy. Journal of experimental \& clinical cancer research. 37, 88 (2018).

15. Mohseni, M., Uludag, H. \& Brandwein, J. M. Advances in biology of acute lymphoblastic leukemia (ALL) and therapeutic implications. American journal of blood research. 8, 29-56 (2018).

16. Jan, R. \& Chaudhry, G. Understanding Apoptosis and Apoptotic Pathways Targeted Cancer Therapeutics. Advanced pharmaceutical bulletin. 9, 205-218 (2019).

17. Pan, S., Li, Z., He, Z., Qiu, J. \& Zhou, S. Molecular mechanisms for tumour resistance to chemotherapy. Clinical and experimental pharmacology \& physiology. 43, 723-737 (2016).

18. Aberuyi, N., Rahgozar, S., Ghodousi, E. S. \& Ghaedi, K. Drug Resistance Biomarkers and Their Clinical Applications in Childhood Acute Lymphoblastic Leukemia. Frontiers in oncology. 9, 1496 (2020).

19. Johnstone, R. W., Ruefli, A. A. \& Lowe, S. W. Apoptosis: A Link between Cancer Genetics and Chemotherapy. Cell. 108, 153-164 (2002).

20. Rodriguez-Lirio, A., Perez-Yarza, G., Fernandez-Suarez, M. R., Alonso-Tejerina, E., Boyano, M. D. \& Asumendi, A. Metformin Induces Cell Cycle Arrest and Apoptosis in Drug-Resistant Leukemia Cells. Leukemia research and treatment. 2015, 516460-12 (2015).

21. Dai, J. \& Mumper, R.J. Plant phenolics: Extraction, analysis and their antioxidant and anticancer properties. Molecules. 15(10), 7313-7352 (2010). 
22. Mohan, A., Narayanan, S., Sethuraman, S. \& Krishnan, U. M. Combinations of Plant Polyphenols \& Anti-Cancer Molecules: A Novel Treatment Strategy for Cancer Chemotherapy. Anti-cancer agents in medicinal chemistry. 13, 281-295 (2013).

23. Montané, X., Kowalczyk, O., Reig-Vano, B., Bajek, A., Roszkowski, K., Tomczyk, R. \& et al. Current Perspectives of the Applications of Polyphenols and Flavonoids in Cancer Therapy. Molecules. 25, 3342 (2020).

24. Sak, K. \& Everaus, H. Established Human Cell Lines as Models to Study Anti-leukemic Effects of Flavonoids. Current genomics. 18, 3-26 (2017).

25. Way, T., Kao, M. \& Lin, J. Degradation of HER2/ neu by apigenin induces apoptosis through cytochrome c release and caspase-3 activation in HER2/ neu-overexpressing breast cancer cells. FEBS letters. 579, 145-152 (2005).

26. Choi, E. J. \& Kim, G. Apigenin Induces Apoptosis through a Mitochondria/Caspase-Pathway in Human Breast Cancer MDA-MB-453 Cells. Journal of clinical biochemistry and nutrition. 44, 260-265 (2009).

27. Choi, E. J. \& Kim, G. Apigenin causes G2/M arrest associated with the modulation of p21Cip1 and Cdc2 and activates p53-dependent apoptosis pathway in human breast cancer SK-BR-3 cells. The Journal of nutritional biochemistry. 20, 285-290 (2009).

28. Chen, D., Landis-Piwowar, K. R., Chen, M. S. \& Dou, Q. P. Inhibition of proteasome activity by the dietary flavonoid apigenin is associated with growth inhibition in cultured breast cancer cells and xenografts. Breast cancer research. 9, R80 (2007).

29. Seo, H., Ku, J. M., Choi, H., Woo, J., Jang, B., Shin, Y. C. \& Ko, S. Induction of caspase-dependent apoptosis by apigenin by inhibiting STAT3 signaling in HER2-overexpressing MDA-MB-453 breast cancer cells. Anticancer research. 34, 2869-2882 (2014).

30. Seo, H., Ku, J. M., Choi, H., Woo, J., Jang, B., Shin, Y. C. \& Ko, S. Apigenin induces caspase-dependent apoptosis by inhibiting signal transducer and activator of transcription 3 signaling in HER2overexpressing SKBR3 breast cancer cells. Molecular medicine reports. 12, 2977-2984 (2015).

31. Kaur, P., Shukla, S. \& Gupta, S. Plant flavonoid apigenin inactivates Akt to trigger apoptosis in human prostate cancer: an in vitro and in vivo study. Carcinogenesis (New York). 29, 2210-2217 (2008).

32. Seo, Y. J., Kim, B. S., Chun, S. Y., Park, Y. K., Kang, K. S. \& Kwon, T. G. Apoptotic effects of genistein, biochanin-A and apigenin on LNCaP and PC-3 cells by p21 through transcriptional inhibition of pololike kinase-1. Journal of Korean medical science. 26, 1489-1494 (2011).

33. Shukla, S., Fu, P. \& Gupta, S. Apigenin induces apoptosis by targeting inhibitor of apoptosis proteins and Ku70-Bax interaction in prostate cancer. Apoptosis. 19, 883-894 (2014).

34. Turktekin, M., Konac, E., Onen, H. I., Alp, E., Yilmaz, A. \& Menevse, S. Evaluation of the Effects of the Flavonoid Apigenin on Apoptotic Pathway Gene Expression on the Colon Cancer Cell Line (HT29). Journal of medicinal food. 14, 117-1117 (2011).

35. Wang, Q. R., Yao, X. Q., Wen, G., Fan, Q., Li, Y., Fu, X. Q., Li, C. K. \& Sun, X. G. Apigenin suppresses the growth of colorectal cancer xenografts via phosphorylation and up-regulated FADD expression. 
Oncology letters. 2, 43-47 (2011).

36. Lee, Y., Sung, B., Kang, Y. J., Kim, D. H., Jang, J., Hwang, S. Y. \& et al. Apigenin-induced apoptosis is enhanced by inhibition of autophagy formation in HCT116 human colon cancer cells. International journal of oncology. 44, 1599-1606 (2014).

37. Wang, B. \& Zhao, X. Apigenin induces both intrinsic and extrinsic pathways of apoptosis in human colon carcinoma HCT-116 cells. Oncology reports. 37, 1132-1140 (2017).

38. Subhasitanont, P., Chokchaichamnankit, D., Chiablaem, K., Keeratichamroen, S., Ngiwsara, L., Paricharttanakul, N. M. \& et al. Apigenin inhibits growth and induces apoptosis in human cholangiocarcinoma cells. Oncology letters. 14, 4361-4371 (2017).

39. Zhu, Y., Mao, Y., Chen, H., Lin, Y., Hu, Z., Wu, J. \& et al. Apigenin promotes apoptosis, inhibits invasion and induces cell cycle arrest of T24 human bladder cancer cells. Cancer cell international. 13, 54 (2013).

40. Shi, M., Shiao, C., Lee, Y. \& Shih, Y. Apigenin, a dietary flavonoid, inhibits proliferation of human bladder cancer T-24 cells via blocking cell cycle progression and inducing apoptosis. Cancer cell international. 15, 33 (2015).

41. Souza, R. P., Bonfim-Mendonça, P. d. S., Gimenes, F., Ratti, B. A., Kaplum, V., Bruschi, M. L. \& et al. Oxidative Stress Triggered by Apigenin Induces Apoptosis in a Comprehensive Panel of Human Cervical Cancer-Derived Cell Lines. Oxidative medicine and cellular longevity. 2017, 1512745-18 (2017).

42. Kayacan, S., Yilancioglu, K., Akdemir, A. S., Dagistanli, F. K., Melikoglu, G. \& Ozturk, M. The Effects of Apigenin and Curcumin on Autophagy Related Cell Death and Apoptosis. Proceedings. 2, 1586 (2018).

43. Lu, H., Chie, Y., Yang, M., Lu, K., Fu, J., Yang, J. \& et al. Apigenin induces apoptosis in human lung cancer $\mathrm{H} 460$ cells through caspase- and mitochondria-dependent pathways. Human \& experimental toxicology. 30, 1053-1061 (2011).

44. Zhu, H., Jin, H., Pi, J., Bai, H., Yang, F., Wu, C. \& et al. Apigenin induced apoptosis in esophageal carcinoma cells by destruction membrane structures. Scanning. 38, 322-328 (2016).

45. Choi, S. I., Jeong, C. S., Cho, S. Y. \& Lee, Y. S. Mechanism of apoptosis induced by apigenin in HepG2 human hepatoma cells: involvement of reactive oxygen species generated by NADPH oxidase. Archives of pharmacal research. 30, 1328-1335 (2007).

46. King, J. C., Li, A., Reber, H. A., Go, V. W., Eibl, G. \& Hines, O. J. Apigenin Induces Pancreatic Cancer Cell Apoptosis by a P53-Mediated Mechanism. The Journal of surgical research. 158, 393 (2010).

47. Lin, C., Chuang, Y., Yu, C., Yang, J., Lu, C., Chiang, J. \& et al. Apigenin Induces Apoptosis through Mitochondrial Dysfunction in U-2 OS Human Osteosarcoma Cells and Inhibits Osteosarcoma Xenograft Tumor Growth in Vivo. Journal of agricultural and food chemistry. 60, 11395-11402 (2012).

48. Stump, T. A., Santee, B. N., Williams, L. P., Kunze, R. A., Heinze, C. E., Huseman, E. D. \& et al. The antiproliferative and apoptotic effects of apigenin on glioblastoma cells. Journal of pharmacy and 
pharmacology. 69, 907-916 (2017).

49. Zhao, G., Han, X., Cheng, W., Ni, J., Zhang, Y., Lin, J. \& Song, Z. Apigenin inhibits proliferation and invasion, and induces apoptosis and cell cycle arrest in human melanoma cells. Oncology reports. 37, 2277-2285 (2017).

50. Hussain, A. R., Khan, A. S., Ahmed, S. O., Ahmed, M., Platanias, L. C., Al-Kuraya, K. S. \& Uddin, S. Apigenin induces apoptosis via downregulation of S-phase kinase-associated protein 2-mediated induction of p27Kip1 in primary effusion lymphoma cells. Cell proliferation. 43, 170-183 (2010).

51. Wang, I., Lin-Shiau, S. \& Lin, J. Induction of apoptosis by apigenin and related flavonoids through cytochrome c release and activation of caspase-9 and caspase-3 in leukaemia HL-60 cells. European journal of cancer. 35, 1517-1525 (1999).

52. Vargo, M. A., Voss, O. H., Poustka, F., Cardounel, A. J., Grotewold, E. \& Doseff, A. I. Apigenin-inducedapoptosis is mediated by the activation of PKC $\delta$ and caspases in leukemia cells. Biochemical pharmacology. 72, 681-692 (2006).

53. Gonzalez-Mejia, M. E., Voss, O. H., Murnan, E. J. \& Doseff, A. I. Apigenin-induced apoptosis of leukemia cells is mediated by a bimodal and differentially regulated residue-specific phosphorylation of heat-shock protein-27. Cell death \& disease. 1, e64 (2010).

54. Budhraja, A., Gao, N., Zhang, Z., Son, Y., Cheng, S., Wang, X., Ding, S. \& et al. Apigenin Induces Apoptosis in Human Leukemia Cells and Exhibits Anti-Leukemic Activity In Vivo. Molecular cancer therapeutics. 11, 132-142 (2012).

55. Mahbub, A. A., Maitre, C. L. L., Haywood-Small, S., Cross, N. A. \& Jordan-Mahy, N. Differential Effects of Polyphenols on Proliferation and Apoptosis in Human Myeloid and Lymphoid Leukemia Cell Lines. Anti-cancer agents in medicinal chemistry. 13, 1601-1613 (2013).

56. Florea, A. \& Büsselberg, D. Cisplatin as an anti-tumor drug: cellular mechanisms of activity, drug resistance and induced side effects. Cancers. 3, 1351-1371 (2011).

57. Choi, E.J. \& Kim, G. 5-Fluorouracil combined with apigenin enhances anticancer activity through induction of apoptosis in human breast cancer MDA-MB-453 cells. Oncology reports. 22, 1533-1537 (2009).

58. Chan, L., Chou, T., Ding, H., Chen, P., Chiang, F., Kuo, P. \& Liang, C. Apigenin induces apoptosis via tumor necrosis factor receptor- and Bcl-2-mediated pathway and enhances susceptibility of head and neck squamous cell carcinoma to 5-fluorouracil and cisplatin. Biochimica et biophysica acta. 1820, 1081-1091 (2012).

59. Xu, Y., Xin, Y., Diao, Y., Lu, C., Fu, J., Luo, L. \& Yin, Z. Synergistic Effects of Apigenin and Paclitaxel on Apoptosis of Cancer Cells. PloS one. 6, e29169 (2011).

60. Shao, H., Jing, K., Mahmoud, E., Huang, H., Fang, X. \& Yu, C. Apigenin sensitizes colon cancer cells to anti-tumor activity of ABT-263. Molecular cancer therapeutics. 12, (2013).

61. Liu, R., Ji, P., Liu, B., Qiao, H., Wang, X., Zhou, L., Deng, T. \& Ba, Y. Apigenin enhances the cisplatin cytotoxic effect through p53-modulated apoptosis. Oncology letters. 13, 1024-1030 (2017). 
62. Huang, S., Yu, M., Shi, N., Zhou, Y., Li, F., Li, X., Huang, X. \& Jin, J. Apigenin and Abivertinib, a novel BTK inhibitor synergize to inhibit diffuse large B-cell lymphoma in vivo and vitro. Journal of Cancer. 11, 2123-2132 (2020).

63. Mahbub, A. A., Le Maitre, C. L., Haywood-Small, S. L., Cross, N. A. \& Jordan-Mahy, N. Polyphenols act synergistically with doxorubicin and etoposide in leukaemia cell lines. Cell death discovery. 1, 15043 (2015).

64. Mahbub, A. A., Maitre, C. L. L., Haywood-Small, S., Cross, N. A. \& Jordan-Mahy, N. Polyphenols enhance the activity of alkylating agents in leukaemia cell lines. Oncotarget. 10, 4570-4586 (2019).

65. Yoo, S. H., Yoon, Y. G., Lee, J. S., Song, Y. S., Oh, J. S., Park, B. S. \& et al. Etoposide induces a mixed type of programmed cell death and overcomes the resistance conferred by Bcl-2 in Hep3B hepatoma cells. International journal of oncology. 41, 1443-1454 (2012).

66. Rao, X., Huang, X., Zhou, Z. \& Lin, X. An improvement of the $2^{\wedge}$ (-delta delta CT) method for quantitative real-time polymerase chain reaction data analysis. Biostatistics, bioinformatics and biomathematics. 3, 71-85 (2013).

67. Ruela-de-Sousa, R. R., Fuhler, G. M., Blom, N., Ferreira, C. V., Aoyama, H. \& Peppelenbosch, M. P. Cytotoxicity of apigenin on leukemia cell lines: implications for prevention and therapy. Cell death \& disease. 1, e19 (2010).

68. Pommier, Y., Sordet, O., Antony, S., Hayward, R. L. \& Kohn, K. W. Apoptosis defects and chemotherapy resistance: molecular interaction maps and networks. Oncogene. 23, 2934-2949 (2004).

69. Cassier, P. A., Castets, M., Belhabri, A. \& Vey, N. Targeting apoptosis in acute myeloid leukaemia. British journal of cancer. 117, 1089-1098 (2017).

70. Horinaka, M., Yoshida, T., Shiraishi, T., Nakata, S., Wakada, M. \& Sakai, T. The dietary flavonoid apigenin sensitizes malignant tumor cells to tumor necrosis factor-related apoptosis-inducing ligand. Molecular cancer therapeutics. 5, 945-951 (2006).

71. Chen, X., Xu, H., Yu, X., Wang, X., Zhu, X. \& Xu, X. Apigenin inhibits in vitro and in vivo tumorigenesis in cisplatin-resistant colon cancer cells by inducing autophagy, programmed cell death and targeting m-TOR/PI3K/Akt signalling pathway. Journal of B.U.ON.: official journal of the Balkan Union of Oncology. 24, 488-493 (2019).

72. Liu, Z., Ding, Y., Ye, N., Wild, C., Chen, H. \& Zhou, J. Direct Activation of Bax Protein for Cancer Therapy. Medicinal research reviews. 36, 313-341 (2016).

73. Walensky, L. D. Targeting BAX to drug death directly. Nature chemical biology. 15, 657-665 (2019).

74. Liu, Z., Zhao, X., Zhang, L. \& Pei, B. Cytochrome C inhibits tumor growth and predicts favorable prognosis in clear cell renal cell carcinoma. Oncology letters. 18, 6026-6032 (2019).

75. Franco, R. \& Cidlowski, J. A. Apoptosis and glutathione: beyond an antioxidant. Cell death and differentiation. 16, 1303-1314 (2009).

76. Ledgerwood, E. C. \& Morison, I. M. Targeting the Apoptosome for Cancer Therapy. Clinical cancer research. 15, 420-424 (2009). 
77. Traverso, N., Ricciarelli, R., Nitti, M., Marengo, B., Furfaro, A. L., Pronzato, M. A., Marinari, U. M. \& Domenicotti, C. Role of Glutathione in Cancer Progression and Chemoresistance. Oxidative medicine and cellular longevity. 2013, 972913-10 (2013).

\section{Figures}
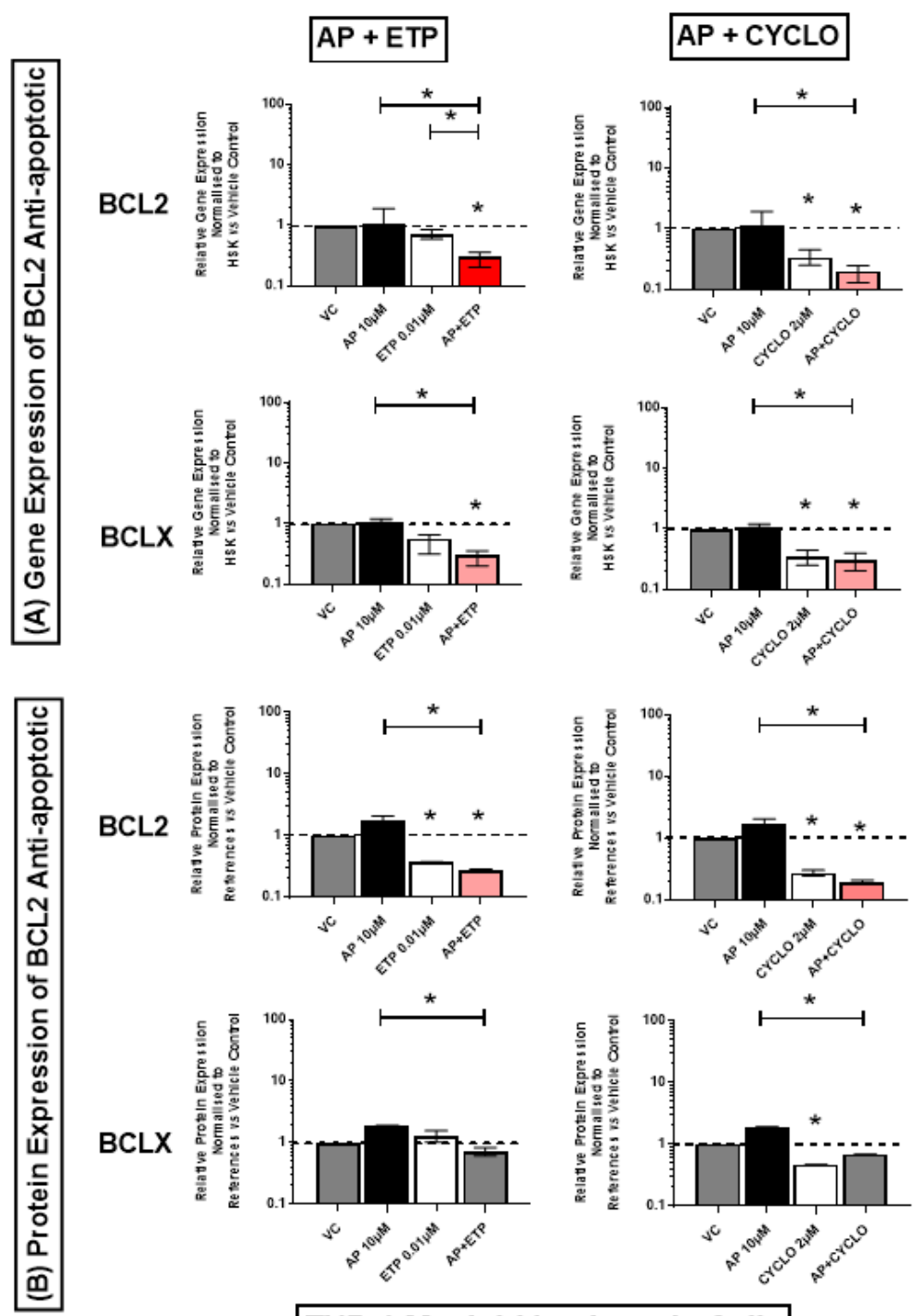

THP-1 Myeloid Leukaemia Cells
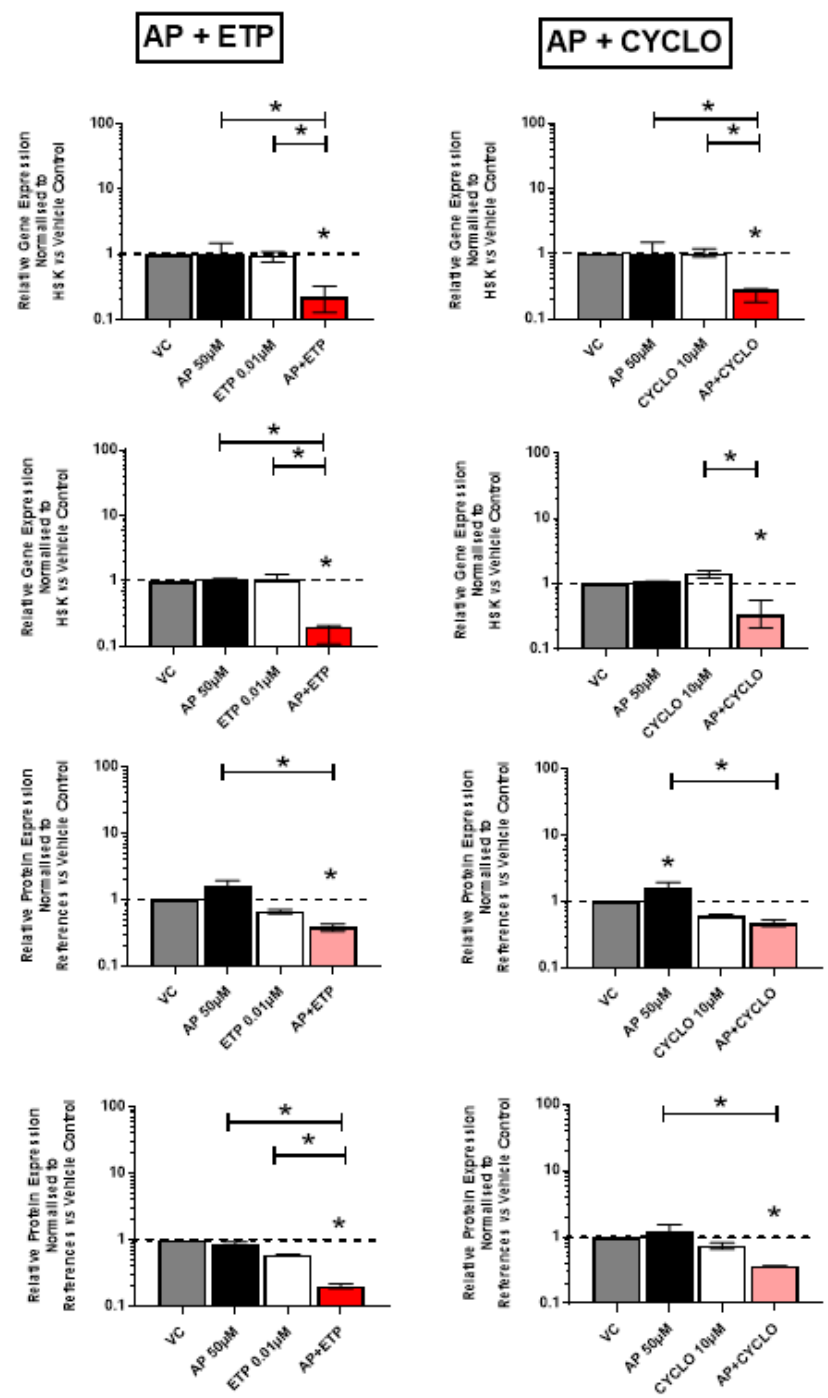

Jurkat Lymphoid Leukaemia Cells

Figure 1

Effects of apigenin (AP) alone and in combination with etoposide (ETP) and cyclophosphamide (CYCLO) on expression of BCL2 and BCLX anti-apoptotic genes (A) and proteins (B) in acute myeloid (THP-1) and lymphoid (Jurkat) leukaemia cell lines when treated with their lowest significant doses (LSDs) that induce apoptosis (determined previously in Mahbub et al., 2013; 2015; 2019) for $24 \mathrm{~h}$. Gene and protein data are expressed as medians and ranges. Results were considered statistically significant when $P \leqslant 0.05\left(^{*}\right)$. The combination treatment effects and colours were determined based on the statistical analysis described in Section 2.4. 

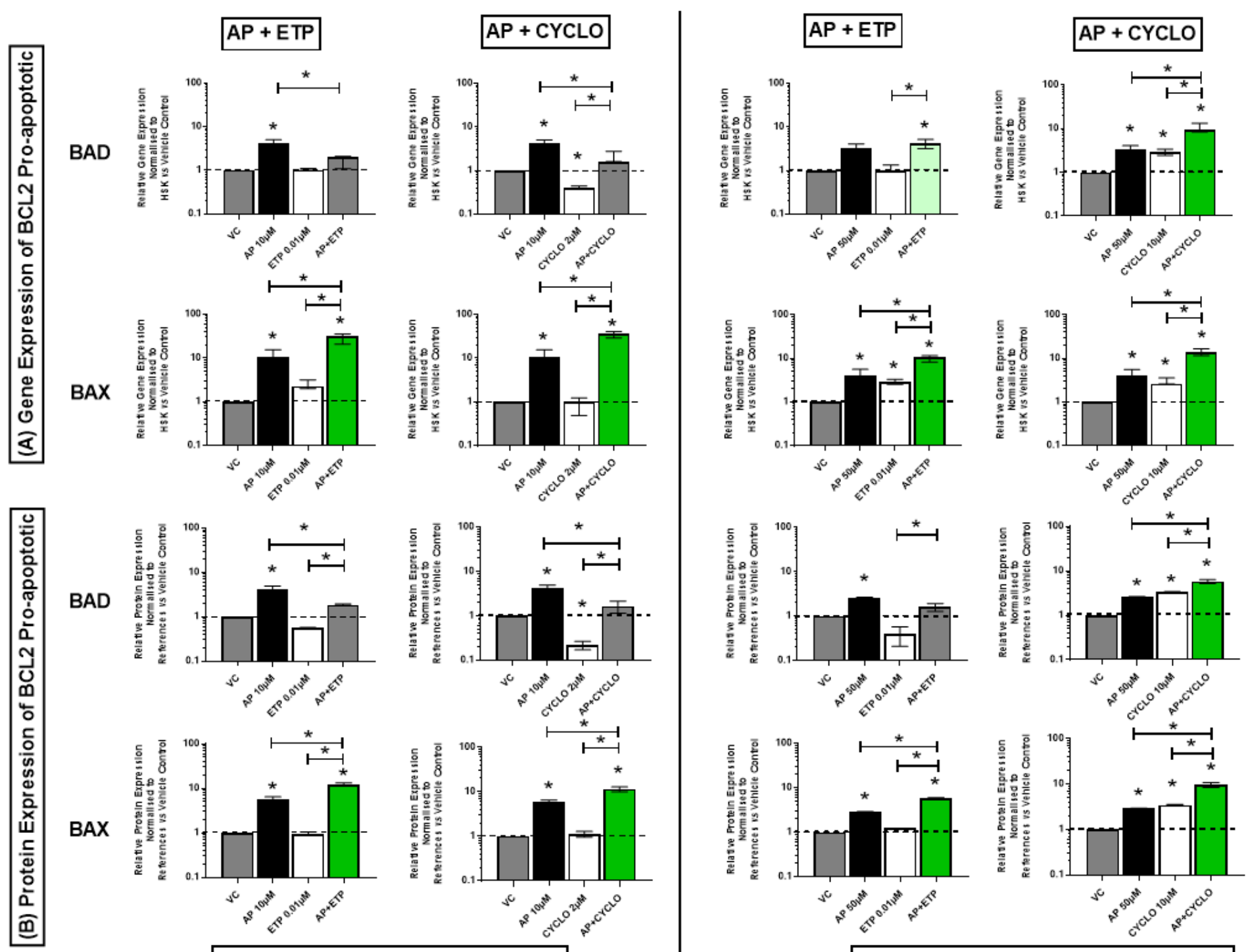

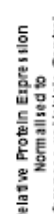
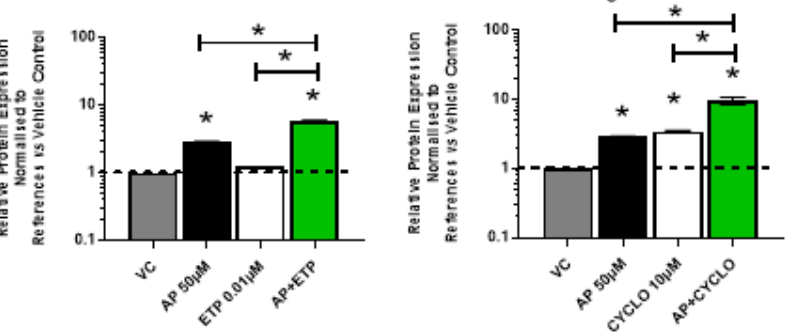

THP-1 Myeloid Leukaemia Cells

Jurkat Lymphoid Leukaemia Cells

Figure 2

Effects of apigenin (AP) alone and in combination with etoposide (ETP) and cyclophosphamide (CYCLO) on expression of BAD and BAX pro-apoptotic genes (A) and proteins (B) in acute myeloid (THP-1) and lymphoid (Jurkat) leukaemia cell lines when treated with their lowest significant doses (LSDs) that induce apoptosis (determined previously in Mahbub et al., 2013; 2015; 2019) for $24 \mathrm{~h}$. Gene and protein data are expressed as medians and ranges. Results were considered statistically significant when $P \leqslant 0.05\left(^{*}\right)$. The combination treatment effects and colours were determined based on the statistical analysis described in Section 2.4. 


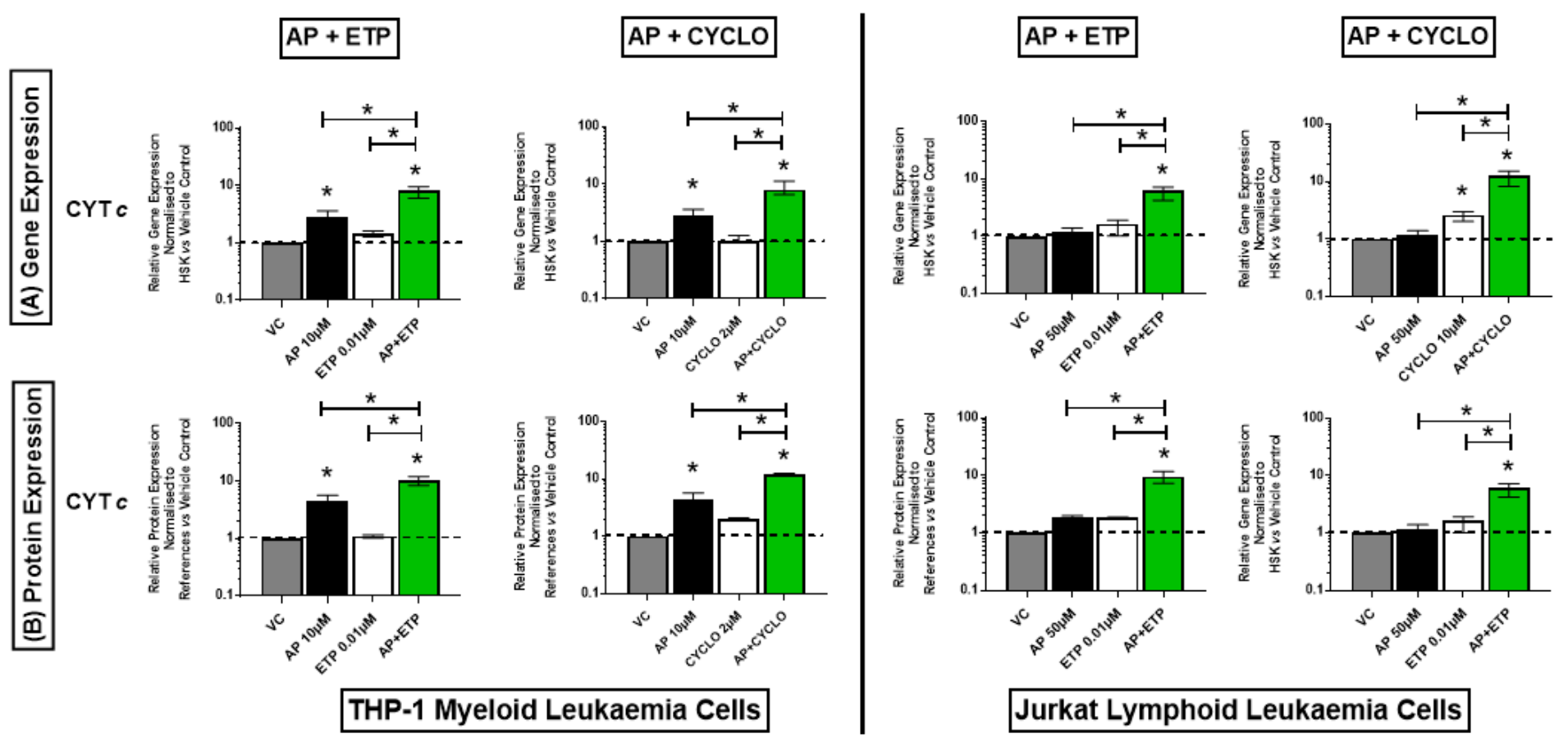

Figure 3

Effects of apigenin (AP) alone and in combination with etoposide (ETP) and cyclophosphamide (CYCLO) on expression of Cytochrome $\mathrm{c}$ (CYT $\mathrm{c}$ ) mitochondrial gene (A) and protein (B) in acute myeloid (THP-1) and lymphoid (Jurkat) leukaemia cell lines when treated with their lowest significant doses (LSDs) that induce apoptosis (determined previously in Mahbub et al., 2013; 2015; 2019) for 24 h. Gene and protein data are expressed as medians and ranges. Results were considered statistically significant when $\mathrm{P}$ $\leqslant 0.05\left(^{\star}\right)$. The combination treatment effects and colours were determined based on the statistical analysis described in Section 2.4. 

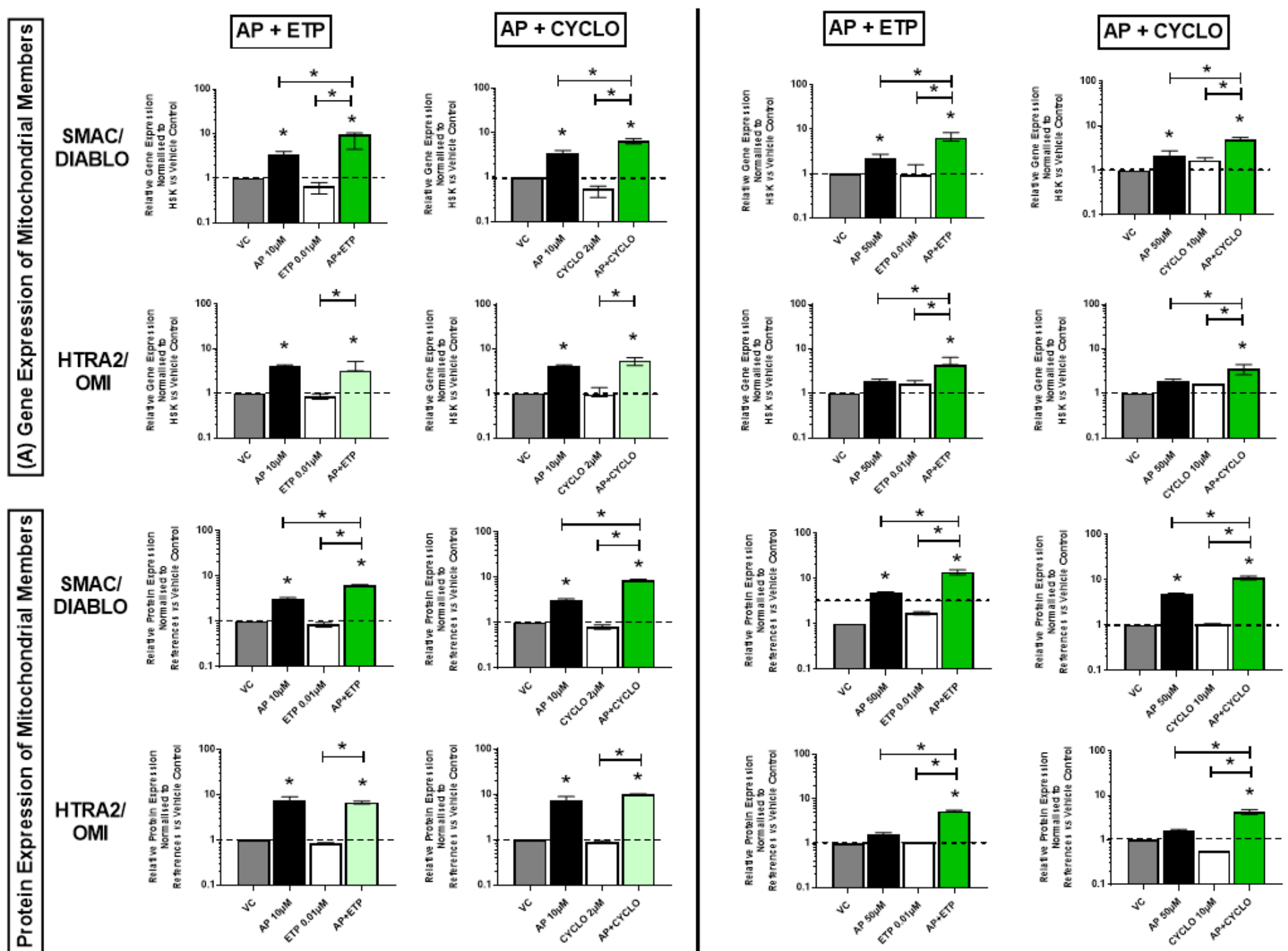

THP-1 Myeloid Leukaemia Cells

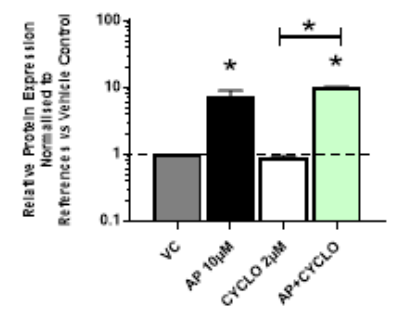

Jurkat Lymphoid Leukaemia Cells

Figure 4

Effects of apigenin (AP) alone and in combination with etoposide (ETP) and cyclophosphamide (CYCLO) on expression of SMAC/ DIABLO and HTRA2/OMI mitochondrial genes (A) and proteins (B) in acute myeloid (THP-1) and lymphoid (Jurkat) leukaemia cell lines when treated with their lowest significant doses (LSDs) that induce apoptosis (determined previously in Mahbub et al., 2013; 2015; 2019 ) for $24 \mathrm{~h}$. Gene and protein data are expressed as medians and ranges. Results were considered statistically significant when $P \leqslant 0.05(*)$. The combination treatment effects and colours were determined based on the statistical analysis described in Section 2.4 . 

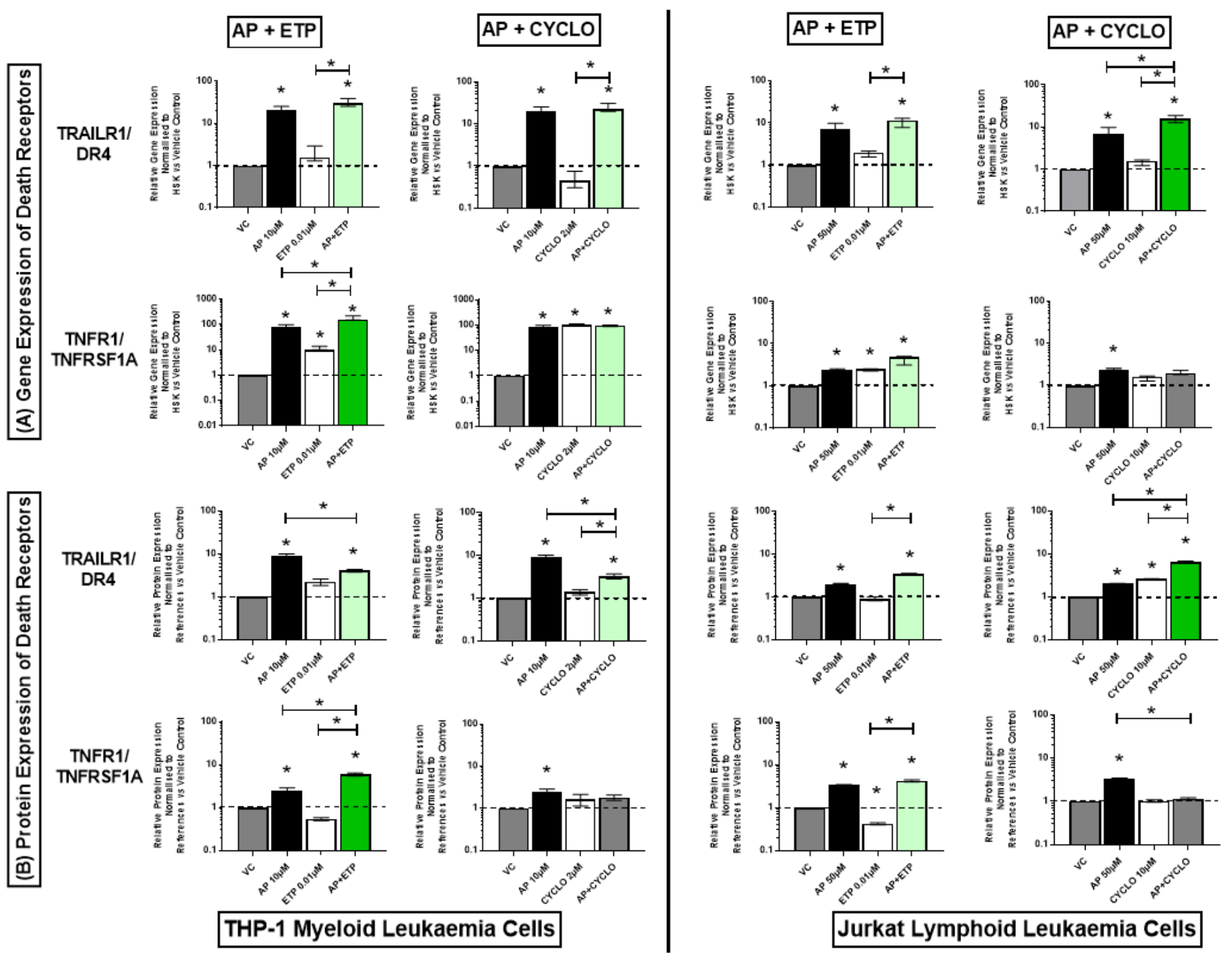

Jurkat Lymphoid Leukaemia Cells

\section{Figure 5}

Effects of apigenin (AP) alone and in combination with etoposide (ETP) and cyclophosphamide (CYCLO) on expression of TRAILR1/DR4 and TNFR1/TNFRSF1A death receptors genes (A) and proteins (B) in acute myeloid (THP-1) and lymphoid (Jurkat) leukaemia cell lines when treated with their lowest significant doses (LSDs) that induce apoptosis (determined previously in Mahbub et al., 2013; 2015; 2019) for $24 \mathrm{~h}$. Gene and protein data are expressed as medians and ranges. Results were considered statistically significant when $P \leqslant 0.05\left(^{*}\right)$. The combination treatment effects and colours were determined based on the statistical analysis as described in Section 2.4. 

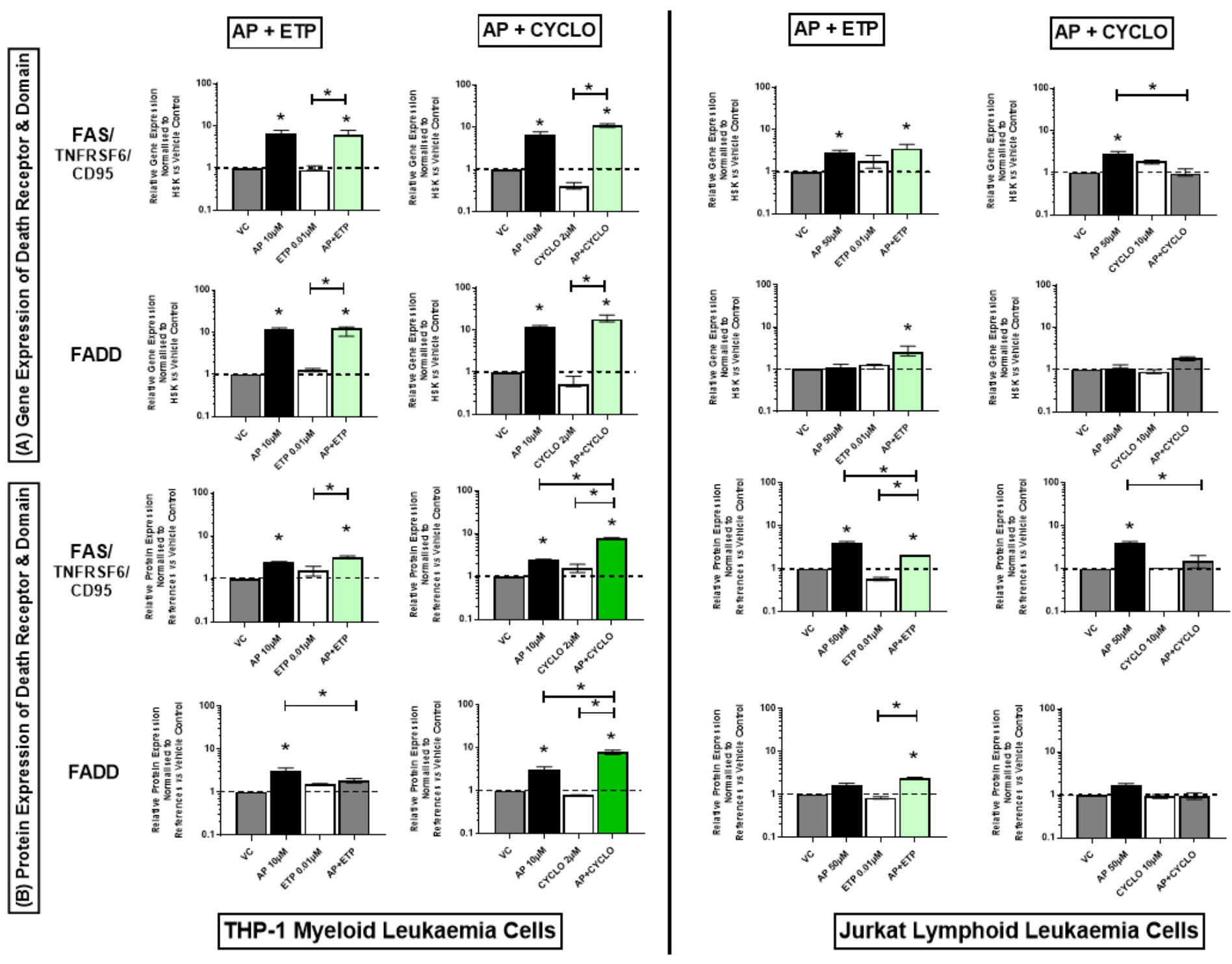

Jurkat Lymphoid Leukaemia Cells

Figure 6

Effects of apigenin (AP) alone and in combination with etoposide (ETP) and cyclophosphamide (CYCLO) on expression of FAS/CD95 death receptor and FADD domain genes $(A)$ and proteins $(B)$ in acute myeloid (THP-1) and lymphoid (Jurkat) leukaemia cell lines when treated with their lowest significant doses (LSDs) that induce apoptosis (determined previously in Mahbub et al., 2013; 2015; 2019) for $24 \mathrm{~h}$. Gene and protein data are expressed as medians and ranges. Results were considered statistically significant when $\left.\mathrm{P} \leqslant 0.05{ }^{*}\right)$. The combination treatment effects and colours were determined based on the statistical analysis as described in Section 2.4. 

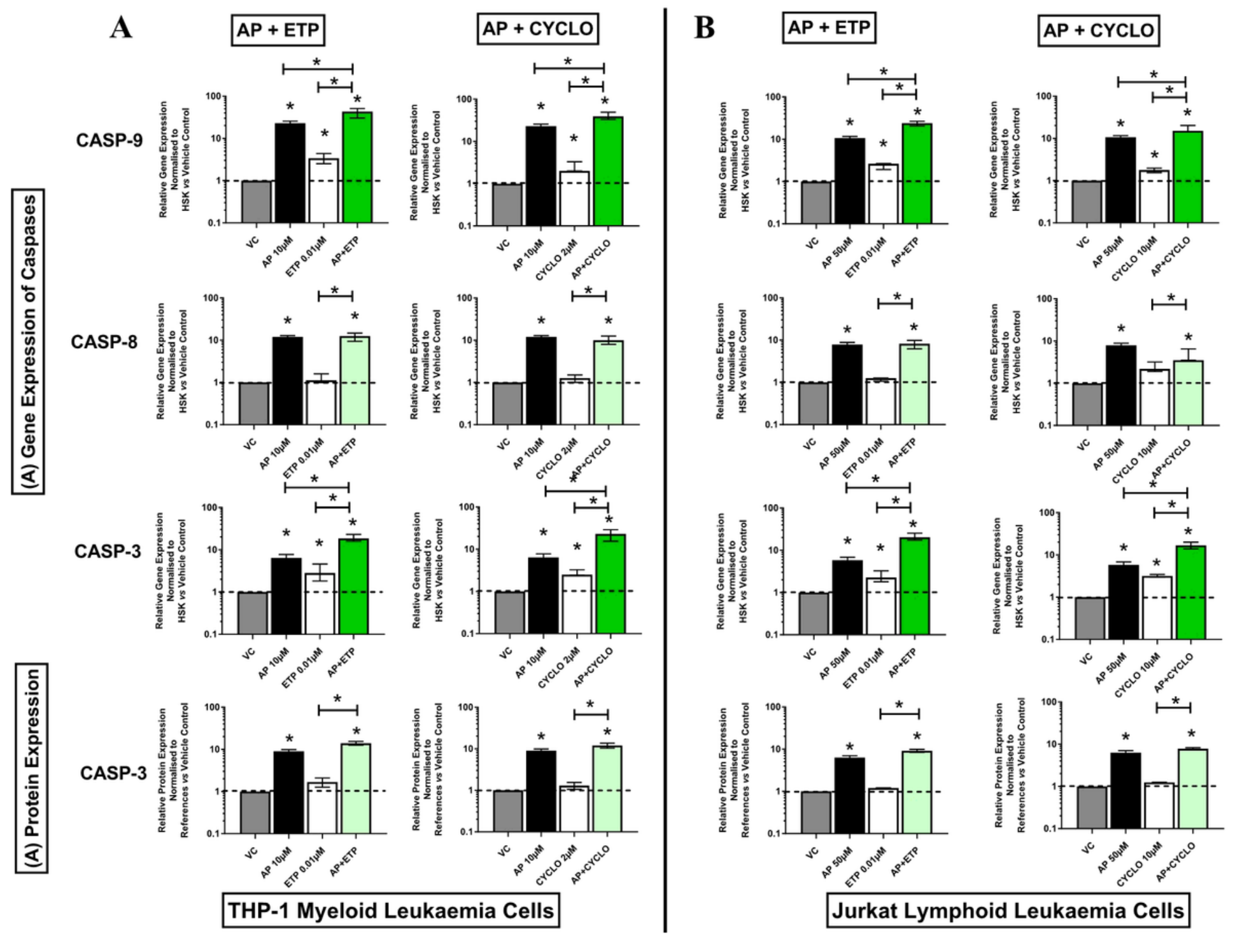

Figure 7

Effects of apigenin (AP) alone and in combination with etoposide (ETP) and cyclophosphamide (CYCLO) on expression of caspase genes (A) and proteins (B) in acute myeloid (THP-1) and lymphoid (Jurkat) leukaemia cell lines when treated with their lowest significant doses (LSDs) that induce apoptosis (determined previously in Mahbub et al., 2013; 2015; 2019) for 24 h. Gene and protein data are expressed as medians and ranges. Results were considered statistically significant when $P \leqslant 0.05(*)$. The combination treatment effects and colours were determined based on the statistical analysis as described in Section 2.4 . 

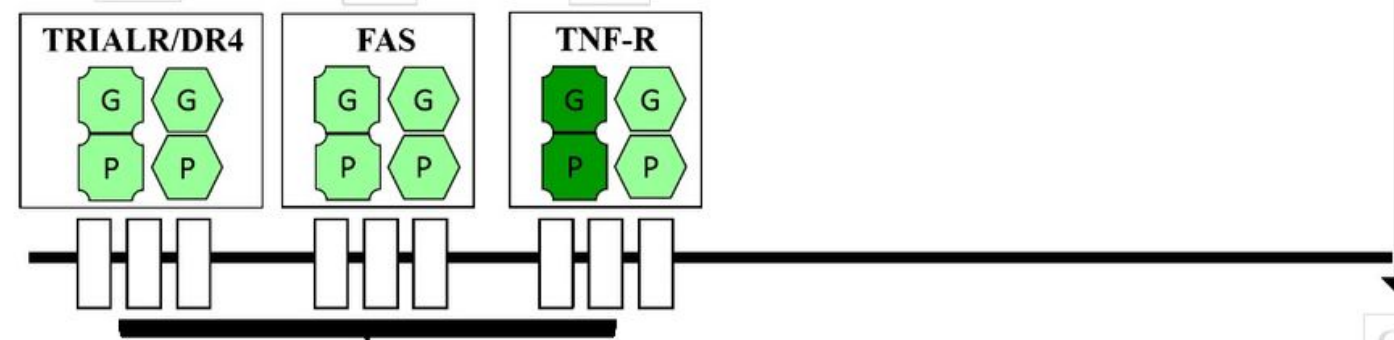

Mitochondrial Pathway (Intrinsic)

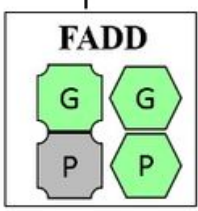

Jurkat lymphoid cells

THP-I mycloid calls

G Gene expression

P Protein expression

Significant increase

Synergistic increase

Significant decrease

Syncrgistic decrease
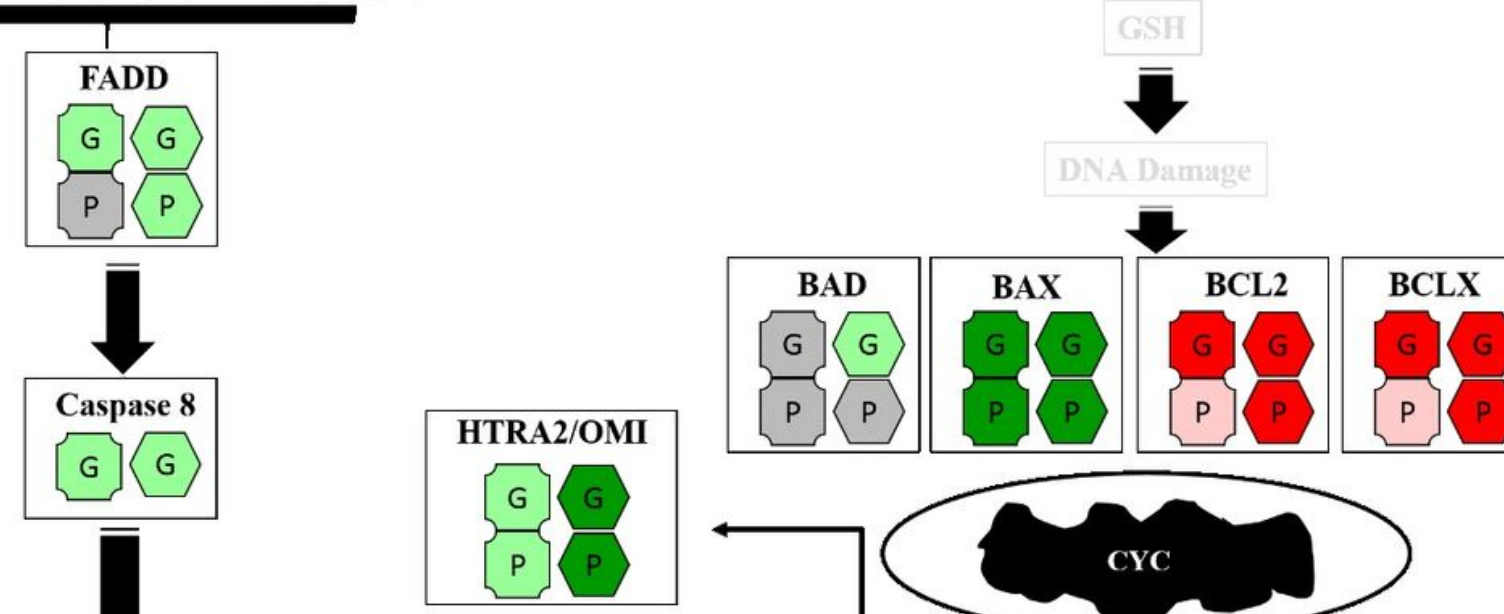

Intracellular
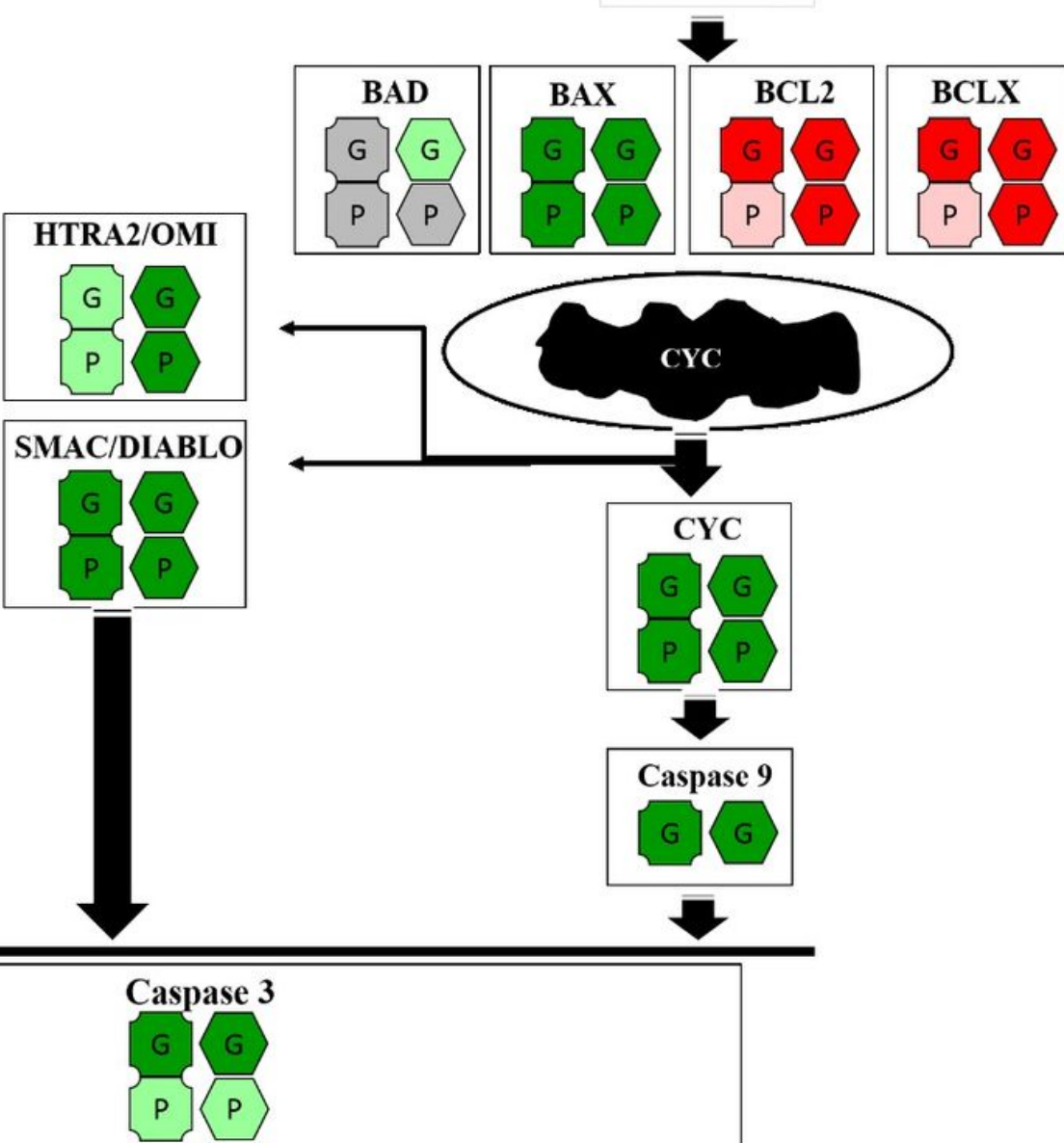

\section{Figure 8}

A summary of the effects of apigenin and etoposide combination treatments on apoptosis-related gene and protein expression in acute leukaemia cells (Jurkat and THP-1). 
(Extrinsic)
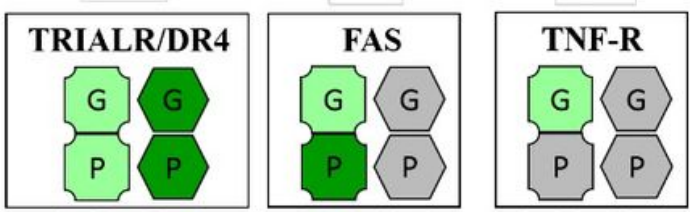
(Intrinsic)
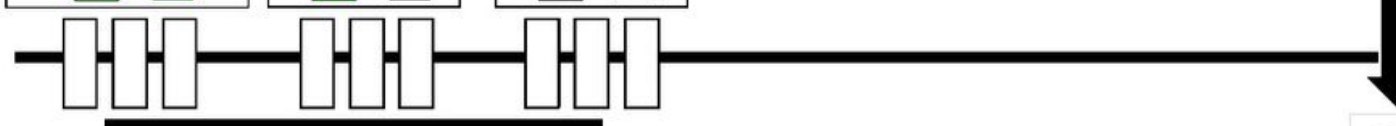

Exrracellalar Intracellular

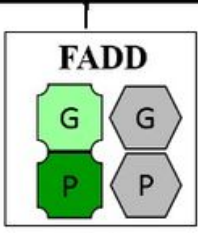

Jurkat lymphoid cells

THP-I myeloid calls

G Genc expression

P Protein expression

Significant increase

Synergislic increase

Significant decrease

Syncrgistic decrease
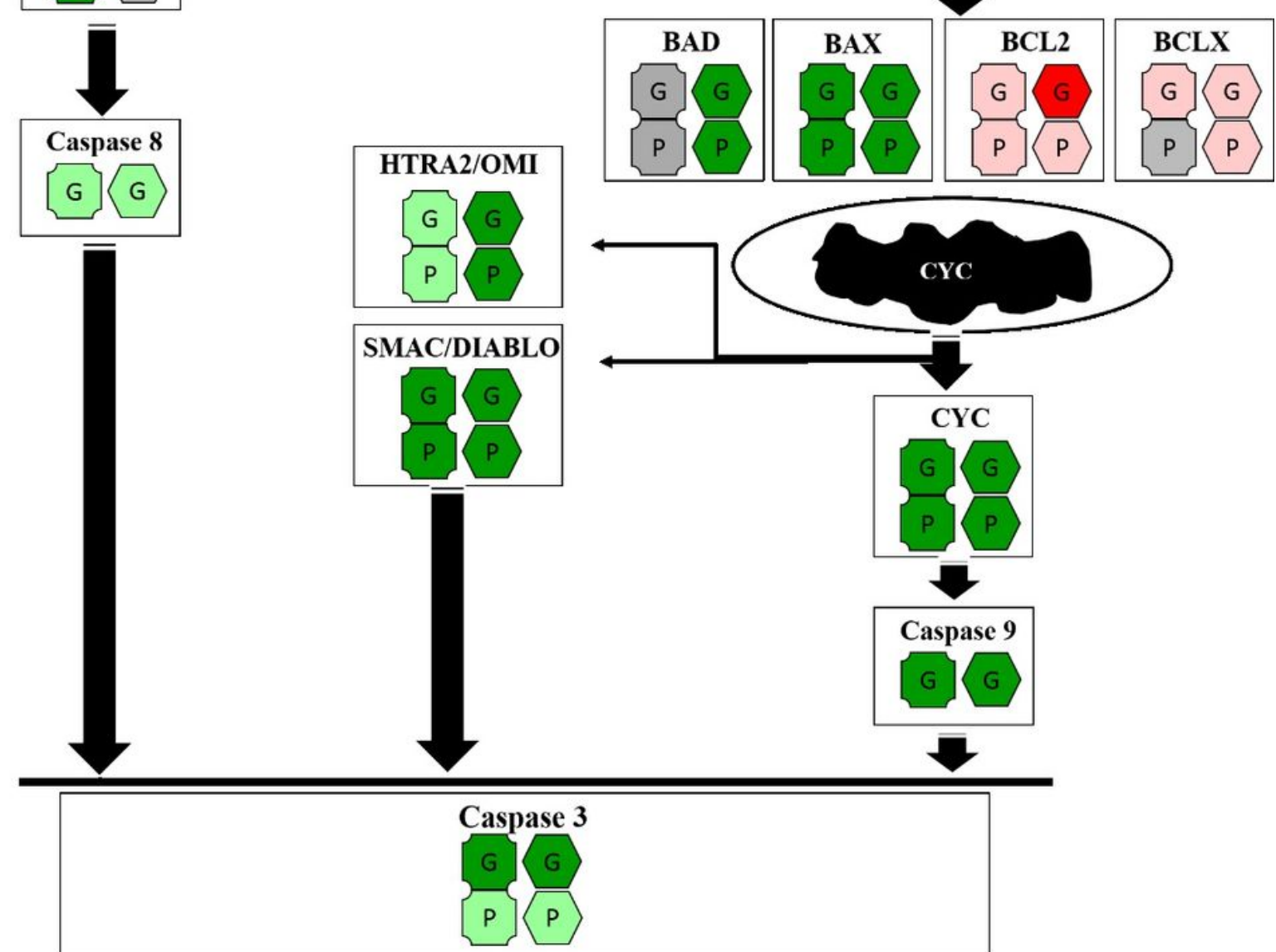

Figure 9

A summary of the effects of apigenin and cyclophosphamide combination treatments on apoptosisrelated gene and protein expression in acute leukaemia cells (Jurkat and THP-1).

\section{Supplementary Files}

This is a list of supplementary files associated with this preprint. Click to download.

- SupplementaryMaterialsonlyFINAL12NOV2021.docx 